\title{
Using RNA-seq to characterize responses to 4-hydroxyphenylpyruvate dioxygenase (HPPD) inhibitor herbicide resistance in waterhemp (Amaranthus tuberculatus)
}

\author{
Daniel R. Kohlhase ${ }^{1}$, Jamie A. O'Rourke ${ }^{2}$, Micheal D. K. Owen ${ }^{*^{*}}$ and Michelle A. Graham ${ }^{2^{*}}$ (D)
}

\begin{abstract}
Background: Waterhemp (Amaranthus tuberculatus (Moq.) J.D. Sauer) is a problem weed commonly found in the Midwestern United States that can cause crippling yield losses for both maize (Zea mays L.) and soybean (Glycine max L. Merr). In 2011, 4-hydroxyphenylpyruvate-dioxygenase (HPPD, EC 1.13.11.27) inhibitor herbicide resistance was first reported in two waterhemp populations. Since the discovery of HPPD-herbicide resistance, studies have identified the mechanism of resistance and described the inheritance of the herbicide resistance. However, no studies have examined genome-wide gene expression changes in response to herbicide treatment in herbicide resistant and susceptible waterhemp.
\end{abstract}

Results: We conducted RNA-sequencing (RNA-seq) analyses of two waterhemp populations (HPPD-herbicide resistant and susceptible), from herbicide-treated and mock-treated leaf samples at three, six, twelve, and twenty-four hours after treatment (HAT). We performed a de novo transcriptome assembly using all sample sequences. Following assessments of our assembly, individual samples were mapped to the de novo transcriptome allowing us to identify transcripts specific to a genotype, herbicide treatment, or time point. Our results indicate that the response of HPPDherbicide resistant and susceptible waterhemp genotypes to HPPD-inhibiting herbicide is rapid, established as soon as 3 hours after herbicide treatment. Further, there was little overlap in gene expression between resistant and susceptible genotypes, highlighting dynamic differences in response to herbicide treatment. In addition, we used stringent analytical methods to identify candidate single nucleotide polymorphisms (SNPS) that distinguish the resistant and susceptible genotypes.

Conclusions: The waterhemp transcriptome, herbicide-responsive genes, and SNPs generated in this study provide valuable tools for future studies by numerous plant science communities. This collection of resources is essential to study and understand herbicide effects on gene expression in resistant and susceptible weeds. Understanding how herbicides impact gene expression could allow us to develop novel approaches for future herbicide development. Additionally, an increased understanding of the prolific traits intrinsic in weed success could lead to crop improvement.

Keywords: RNA-seq, de novo transcriptome assembly, herbicide resistance, HPPD, Amaranthus tuberculatus

\footnotetext{
* Correspondence: mdowen@iastate.edu; michelle.graham@ars.usda.gov

'Department of Agronomy, lowa State University, Ames, IA, USA

${ }^{2}$ U.S. Department of Agriculture (USDA)-Agricultural Research Service (ARS),

Corn Insects and Crop Genetics Research Unit, Ames, IA, USA
}

(c) The Author(s). 2019 Open Access This article is distributed under the terms of the Creative Commons Attribution 4.0 International License (http://creativecommons.org/licenses/by/4.0/), which permits unrestricted use, distribution, and reproduction in any medium, provided you give appropriate credit to the original author(s) and the source, provide a link to the Creative Commons license, and indicate if changes were made. The Creative Commons Public Domain Dedication waiver (http://creativecommons.org/publicdomain/zero/1.0/) applies to the data made available in this article, unless otherwise stated. 


\section{Background}

Over the past 30 years, waterhemp (Amaranthus tuberculatus (Moq.) J.D. Sauer) has evolved into a major problem weed species in agricultural production systems across the Midwestern United States [1]. If not properly managed, fields infested with waterhemp can suffer yield losses up to $74 \%$ in maize (Zea mays L.) and $56 \%$ in soybean (Glycine $\max (\mathrm{L}$.) Merr.) [2, 3]. Waterhemp is native to the Midwestern United States and is dioecious; the male and female reproductive structures are on separate plants. As a dioecious species, waterhemp is an obligatory outcrosser, resulting in high levels of genetic recombination and variability. Obligatory outcrossing facilitates the movement of ecologically valuable traits, such as herbicide resistance, among and between waterhemp populations. Additional biological traits that contribute to the weediness of waterhemp include prolific seed production [4], extended and opportunistic germination [5], and rapid growth rate [6].

Herbicides are currently the most important tool in weed management for most crop production systems in many parts of the world [7]. A major concern of modern weed management is evolved resistance to herbicides. One of the first documented cases of evolved herbicide resistance in weeds was reported in 1970 and since then, the number of unique cases (an individual species $\mathrm{x}$ specific herbicide site of action) has grown to 498 globally and continues to increase $[8,9]$.

In 2011, two waterhemp populations with evolved resistance to 4-hydroxyphenylpyruvate dioxygenase (HPPD, EC 1.13.11.27) inhibiting herbicides, including mesotrione, were discovered in seed maize fields in Iowa and Illinois [10, 11]. Mesotrione (2-(4-Mesyl-2-nitrobenzoyl)-1,3-cyclohexanedione, Herbicide Group (HG) 27) is a selective herbicide that is commonly used for broadleaf weed control in maize [12]. HPPD converts 4-hydroxyphenylpyruvate (4-HPP) to homogentisate (2,5-dihydroxyphenylacetate; HGA), which is an important precursor in carotenoid biosynthesis. The herbicidal activity of mesotrione is characterized by the bleaching of new tissue followed by tissue necrosis. While the resistance mechanisms in the Iowa population have not been determined, in the Illinois population, herbicide resistance was conferred by the metabolism of mesotrione to non-herbicidal metabolites, reportedly attributable to increased cytochrome P450 monooxygenase (CYP P450, EC 1.14.14.1) activity [13]. In 2017, mesotrione resistance was confirmed in two waterhemp populations from Nebraska (Columbus, NE, [14] and Tarnov, NE, [15]). In the Tarnov population, which was used in this study, Kaundun et al. [15] found the HPPD gene had no target site mutations associated with mesotrione resistance, nor was the HPPD gene duplicated or overexpressed. However, they observed increased mesotrione metabolism in the resistant population, again attributed to cytochrome P450 activity. Finding similar resistance mechanisms in distant populations (Nebraska and Illinois) suggests resistance occurs through spontaneous evolution of standing genetic variation [16]. Further, in the HPPD-resistant populations examined thus far [17-19], resistance is polygenic, making identification of causal genes more difficult. None of these studies examined genome wide expression changes in response to mesotrione in resistant and susceptible waterhemp populations. Characterizing gene expression differences in HPPD-resistant and susceptible waterhemp populations at time points well before metabolic responses are detected could help identify major genes contributing to resistance and may provide insight into managing the evolution of resistance to other herbicides in waterhemp and possibly other weed species.

Advances in sequencing technologies have created opportunities to study the genomics of non-model organisms [20]. Due to a lack of weed-related genomic resources, Lee et al. [20] sampled 43 million base pairs of the waterhemp genome using 454 pyrosequencing (Roche Sequencing, Pleasanton, CA, USA). While this sequencing approach covered less than $10 \%$ of the waterhemp genome, it demonstrated that cutting-edge sequencing technology could be applied to weed species. Riggins et al. [21] used 454 pyrosequencing to analyze the waterhemp transcriptome. To maximize transcriptome coverage, the study pooled RNA samples from different individuals, sexes, tissues, life stages, herbicide treatments, and cold stress. These studies contributed to a better understanding of the waterhemp genome and provided sequence-based details for key enzymes targeted by herbicides and potentially prone to herbicide resistance evolution [21]. However, the experimental designs and sequencing platforms used in these studies made it impossible to identify the genes and gene networks that regulate susceptibility, tolerance and resistance to herbicides. Since these initial studies, RNA-sequencing (RNA-seq) has become the standard method for transcriptome analyses for species lacking genomic resources.

The increasing prominence of waterhemp as an economically important and ubiquitous weed in the Midwestern United States and the demonstrated ability to evolve resistance to herbicides makes this species an important model for studying herbicide resistance evolution in weeds. Here we report on the sequencing of the waterhemp transcriptome using high throughput RNA-seq technology. This study identifies the genes and gene networks responding to the HPPD-inhibiting herbicide mesotrione in susceptible and resistant waterhemp genotypes over a 24-h exposure time course. In addition, our study provides a publicly available sequence-based platform for the weed science community to study this agronomically important weed. 


\section{Methods}

\section{Tissue collection}

Two waterhemp populations with different susceptibility phenotypes to HPPD-inhibiting herbicides (susceptible and resistant) were selected. The susceptible waterhemp population was collected from the Curtis Farm at Iowa State University (Ames, IA, USA) in 2006. The resistant population was from Tarnov, Nebraska (USA) and was collected in 2014 from a field with a history of seed maize production after reports of waterhemp surviving multiple applications of mesotrione [15].

Each genotype was planted in 40 individual $15.2 \mathrm{~cm}$ in diameter round pots using a 4:1 ratio of Sunshine potting mix \#1/LC1 (Sun Gro Horticulture, Agawam, MA, USA) to sand, respectively. We added 1 tsp. of Osmocote Flower Food Granules (14-14-14) (The Scotts Miacle-Gro Company, Marysville, OH, USA) to each pot at the time of planting. Plants were grown in a greenhouse set to $24{ }^{\circ} \mathrm{C}$ with a 14 -h photoperiod supplemented by high-pressure sodium bulbs. Plants were watered every other day. After 2 weeks, seedlings were thinned to 3 plants per pot. Each plant within each pot was randomly assigned a label of $\mathrm{A}, \mathrm{B}$, or $\mathrm{C}$. The pots were placed in the greenhouse in a randomized block.

When plants reached a minimum height of $7.6 \mathrm{~cm}$, they were treated with mesotrione applied in a $\mathrm{CO}_{2}$ powered spray chamber equipped with TeeJet ${ }^{\oplus}$ 80015EVS nozzles (Spraying Systems Co., Wheaton, IL, USA) at a carrier volume of $191.76 \mathrm{~L} \mathrm{ha}^{-1}$. Half of each population (20 pots of each genotype) was treated with $105.36 \mathrm{~g}$ ai ha ${ }^{-1}$ of mesotrione, $1 \%(\mathrm{v} / \mathrm{v})$ crop oil concentrate $(\mathrm{COC}), 2.5 \%(\mathrm{v} / \mathrm{v})$ urea $\left(\mathrm{CH}_{4} \mathrm{~N}_{2} \mathrm{O}\right)$ ammonium nitrate $\left(\mathrm{NH}_{4} \mathrm{NO}_{3}\right)$ solution (UAN; $28 \%$ nitrogen), and tap water. The other half was treated with water, representing a mock treatment. The plants were then returned to the greenhouse into 4 blocks separated by treatment and genotype. To determine other reagents used in conjunction with the herbicide affected plant growth, a separate study was used to evaluate the phenotypic response of plants that were untreated, mock-treated, and treated with $1 \%(\mathrm{v} / \mathrm{v})$ COC, $2.5 \%(\mathrm{v} / \mathrm{v})$ UAN (28\% nitrogen), and tap water (data not provided). No difference in phenotypic response was identified.

Each genotype within a treatment was separated into 4 groups of 5 pots. The 4 groups were randomly assigned a time point of $3,6,12$, or 24 HAT. Within each time point 4 pots were labeled $1-4$. The fifth pot was used as a control for verification of the phenotypic response and was also used as a buffer against greenhouse variation in the bench space adjacent to the wall. Leaf tissue from each plant within the four labeled pots was collected at 12:00 PM CDT (3 HAT), 3:00 PM CDT (6 HAT), 9:00 PM CDT (12 HAT), and 9:00 AM CDT (24 HAT) on May 22 and 23 of 2015. Sunrise occurred at 5:49 AM, while sunset occurred at 8:34 PM. A given plant was only sampled at one time point. To obtain the highest quality RNA, the four youngest fully-developed leaves of each plant were excised at the base of each leaf, placed in a $50 \mathrm{~mL}$ Falcon ${ }^{\oplus}$ tube (Thermo Fisher Scientific, Waltham, MA, USA), flash frozen in liquid nitrogen, and then maintained at $-80^{\circ} \mathrm{C}$. Tissues from individual plant samples were stored in a separate Falcon ${ }^{\odot}$ tube. Plants continued to grow for 3 weeks after treatment to verify the phenotypic response to mesotrione.

\section{RNA isolation}

Frozen tissue in the $50 \mathrm{~mL}$ Falcon ${ }^{\circledR}$ tubes was crushed by inverting an $11.11 \mathrm{~cm}$ pestle, dipped in liquid nitrogen, into the tubes. Crushing the leaf samples within a Fal$\operatorname{con}^{\bullet}$ tube mixed the tissue from an individual plant providing a more homogeneous collection of leaves from each plant. One full microspatula scoop (approximately $100 \mathrm{mg}$ ) of crushed frozen tissue from each Falcon ${ }^{\oplus}$ tube was added to a $2 \mathrm{~mL}$ Safe-Lock ${ }^{\mathrm{TM}}$ microcentrifuge tube (Eppendorf, Hamburg, Germany) kept on dry ice with a $3 \mathrm{~mm}$ tungsten carbide bead. Prepared microtubes were placed in TissueLyser Adapter sets precooled at $-80{ }^{\circ} \mathrm{C}$ and then processed in a Qiagen TissueLyser II (Qiagen, Valencia, CA, USA) for $1 \mathrm{~min}$ at $30 \mathrm{~Hz}$. RNA extraction was performed as recommended by the manufacturer using the RNeasy ${ }^{\circ}$ Plant Mini Kit (Qiagen, Valencia, CA, USA). To check for RNA concentration and quality, a NanoDrop $^{\text {тм }} 1000$ Spectrophotometer (Thermo Fisher Scientific, Waltham, MA, USA) was used.

Prior to DNase treatment, to remove genomic DNA contamination, RNA samples from plants growing in the same pot were pooled together. $6 \mu \mathrm{g}$ of pooled RNA ( $2 \mu \mathrm{g}$ of RNA per sample) were used in a $50 \mu \mathrm{L}$ DNase reaction using the Ambion ${ }^{\oplus}$ TURBO DNA-free ${ }^{\mathrm{TM}}$ Kit (Thermo Fisher Scientific, Waltham, MA, USA). Immediately after DNase treatment, samples were further purified using the RNeasy ${ }^{\oplus}$ MinElute ${ }^{\odot}$ Cleanup Kit following the manufacturer's recommendations. RNA concentration and quality of the samples were checked using the NanoDrop ${ }^{\mathrm{TM}} 1000$ Spectrophotometer.

\section{RNA-Seq and de novo transcriptome assembly}

The extracted RNA was sequenced by the Iowa State University DNA Facility using the Illumina HiSeq 2500 (Illumina, Inc., San Diego, CA, USA) platform. Prior to sequencing, the quality of all samples was confirmed using an Agilent $^{\circ} 2100$ Bioanalyzer $^{\mathrm{TM}}$ (Agilent ${ }^{\circ}$, Santa Clara, CA). RNA was considered acceptable if the RNA integrity number (RIN) was greater than seven. Sequences were generated in High Output Mode with 100 base pair read length and paired-end sequencing. The paired-end protocol, sequencing the RNA from both directions of the strand, enables better transcriptome 
coverage. Forty-eight samples were run on one eightlane flow cell, six samples per lane. Each lane contained three samples of each treatment (herbicide or mock), of one waterhemp genotype (resistant or susceptible), at one time point $(3,6,12$, or $24 \mathrm{HAT})$.

The programs Scythe [22] and Sickle [23] were used to remove sequencing artifacts, low-quality bases $(\mathrm{q}<20)$ and short reads $(1<50)$ from all 48 sequenced samples. Trinity (version 2.0.6, [24]) was used to produce multiple de novo transcriptome assemblies. Three separate assemblies (versions 1-3) were made using kmer lengths of 25, 29, and 32 (Additional file 1). After comparing assembly statistics (total number of transcripts, contig N50, median contig length, and average contig size), version 3 (kmer length 32) was selected because this assembly resulted in the longest N50 (Table 1). However, assembly version 3 still contained contigs that lacked open reading frames or were expressed at very low levels. Therefore, in order to create an improved assembly that could be used for measuring differential gene expression, this assembly was processed with three additional steps. First, the TransDecoder utility within Trinity [25] was used to return transcripts that contained an open reading frame (ORF) of at least 100 amino acids. Second, the program kallisto [26] was used to estimate the number of counts per transcript. Any transcripts with less than 10 counts were removed. Finally, BLASTN analyses (E-value cutoff of $\mathrm{E}<10^{-20}$, [27]) was used to compare the v3 assembly to predicted transcripts in the sugar beet genome (Beta vulgaris L., Refbeet v1.2, [28]), representative species of the ten plant clades of Phytozome (version 10, [29]), and all sequences available in the GenBank nucleotide (NT) database (version 1/22/2016, [30]). Any transcript that was best matched to a non-plant species or had no significant hits was not included in the final assembly. These filtering steps resulted in the final transcriptome, version 4 (v4, Additional file 2).

\section{Functional annotation}

The v4 transcriptome was annotated (Additional file 3) using BLASTX $\left(\mathrm{E}<10^{-10},[27]\right)$ against proteins from Arabidopsis thaliana (The Arabidopsis Information Resource version 10 [TAIR10], [31]), sugar beet (Refbeet v1.2, [28]) and Uniref100 (version 1/22/2016, [32]) and using BLASTN $\left(\mathrm{E}<10^{-20},[27]\right)$ against nucleotides from grain amaranth (Phytozome v12.1, Amaranthus hypochondriacus v2.1, [33]). Custom Perl scripts were used to assign gene ontology (GO) biological processes and molecular function terms [34] based on the top A. thaliana hit. To measure the breadth of the de novo v4 transcriptome relative to related species with complete genome sequences, predicted proteins from poplar (Phytozome v12.1.6, Populus trichocarpa v3.1, [35]), papaya (Phytozome v12.1.6, Carica papaya ASGPBv0.4, [36]), asparagus (Phytozome v12.1.6, Asparagus officinalis V1.1, [37]), sugar beet (Refbeet v1.2, [28]), and grain amaranth (Phytozome v12.1, Amaranthus hypochondriacus v2.1, [33]) genomes were also compared to A. thaliana (TAIR version 10, [31]) using BLASTP $\left(\mathrm{E}<10^{-10}\right.$, [27]). Custom Perl scripts were then used to assign GO biological process and GO slim information based on the best $A$. thaliana homolog. Within each species, the total number of each GO slim count was divided by the total count of all GO slims to adjust for genome duplications.

\section{Differential expression analyses}

The individual sample reads were mapped to the version 4 transcriptome assembly using Bowtie [38]. RNA-seq by Expectation-Maximization (RSEM) software [39] was used to account for reads that could re-align to multiple assembled transcripts in the de novo assembly due to alternatively spliced isoforms. The raw expression counts were normalized across samples using the Trimmed Mean of M-values (TMM) method [40] in edgeR [41]. GGplot2 (CRAN, [42]) was used to compare and visualize read

Table 1 Comparison of waterhemp transcriptome assemblies

\begin{tabular}{|c|c|c|c|c|c|}
\hline & & v1 (k-mer = 25) & v2 $(\mathrm{k}-\mathrm{mer}=29)$ & v3 $(\mathrm{k}-\mathrm{mer}=32)$ & v4 \\
\hline & Total 'gene' count & 269,388 & 238,782 & 226,402 & 42,040 \\
\hline & Total transcript count & 512,945 & 471,767 & 451,199 & 113,893 \\
\hline \multirow[t]{3}{*}{ All Transcripts } & Contig N50 & 762 & 926 & 1029 & 1709 \\
\hline & Median Contig Length & 390 & 424 & 448 & 1094 \\
\hline & Average Contig Length & 598.54 & 669.21 & 713.94 & 1317.45 \\
\hline \multirow[t]{3}{*}{ Longest Isoform } & Contig N50 & 662 & 747 & 792 & 1816 \\
\hline & Median Contig Length & 359 & 371 & 375 & 998 \\
\hline & Average Contig Length & 549.32 & 590.09 & 609.47 & 1301.70 \\
\hline
\end{tabular}

Trinity (version 2.0.6, [24]) was used to generate three unique de novo waterhemp transcriptome assemblies: v1, v2, and v3 based on different kmer length requirements. With each assembly, the number of total transcripts decreased while average contig length increased (see Additional file 1). A total of 2.3 billion reads representing different genotypes, treatments and time points were used in the assembly. The v4 assembly is a subset of the v3 assembly, with transcripts that were redundant, lacking open reading frames or expressed at low levels removed 
counts across replicate samples for technical reproducibility. Transcripts with a log count per million less than one $(\log \mathrm{CPM}<1)$ across all samples were excluded from the analyses, leaving 72,697 expressed transcripts ( $\mathrm{v} 4$ isoforms). edgeR was also used to identify significantly (false discovery rate $(F D R)<0.05$, [43]) differentially expressed transcripts (DETs, Table 2, Additional file 4) responding to treatment (mesotrione treatment vs. mock) in each genotype at each time point (R3, R6, R12, R24, S3, S6, S12 and S24) and across all time points ( $\mathrm{R}$ and $\mathrm{S}$ ).

Overrepresented GO terms associated with DETs of interest were identified using a Fisher's exact test [44] to compare the number of times each GO term was found within a list DETs of interest relative to the number of times each GO term was found among all transcripts in the $\mathrm{v} 4$ assembly (Additional file 5). A Bonferroni correction $(P<0.05,[45])$ was applied to correct for over testing.

\section{Clustering of herbicide-responsive DETs}

To determine if herbicide-responsive DETs might physically cluster in the genome, we took advantage of the closely related grain amaranth genome [33]. BLASTN $\left(\mathrm{E}<10^{-20}\right.$, [27]) was used to compare waterhemp transcripts to predicted transcripts from the grain amaranth genome (Phytozome v12.1, Amaranthus hypochondriacus v2.1, [33]). BLASTN was then used to compare the top grain amaranth hit $\left(\mathrm{E}<10^{-20},[27]\right)$ back to all waterhemp transcripts. Waterhemp transcripts were considered orthologous to grain amaranth transcripts if reciprocal BLAST identified any of the original waterhemp transcript isoforms as a top hit. Custom Perl scripts were used to identify the genomic location of the orthologous grain amaranth transcript from the general feature format (GFF) file corresponding to grain amaranth genome.

A window size of $100,000 \mathrm{bp}$, centered on transcription start sites, was used to identify clusters of DETs. DETs that overlapped by $\pm 50,000$ bp from transcription start sites were considered part of the same cluster. Start site positions from transcripts in the same cluster were

Table 2 Summary of the differentially expressed transcripts (DETs) responding to mesotrione treatment in waterhemp

\begin{tabular}{llllll}
\hline Genotype & \multicolumn{4}{l}{ Hours After Treatment } & \\
\cline { 2 - 5 } & 3 & 6 & 12 & 24 & Across Time \\
\hline Resistant & 89 & 62 & 61 & 1983 & 2091 \\
Susceptible & 500 & 77 & 61 & 565 & 1246 \\
Overlap & 42 & 5 & 9 & 83 & 330 \\
Percent Overlap & $7.7 \%$ & $3.7 \%$ & $8.0 \%$ & $3.4 \%$ & $11.0 \%$
\end{tabular}

DETs were identified at 3, 6, 12, and $24 \mathrm{~h}$ after treatment (HAT) in the resistant and susceptible waterhemp genotypes. Percent overlap between genotypes at a specific time point was calculated by dividing the number of DETs in common between genotypes by the total number of unique DETs at used to calculate an average position for the cluster. Only clusters with four or more DETs are reported (Additional file 6). For clustering, multiple isoforms mapping to the same cluster were considered a single DET transcript.

\section{Identification of candidate single nucleotide polymorphisms between resistant and susceptible waterhemp genotypes}

The sequence alignment files generated for differential expression analysis were sorted using samtools and then merged by genotype into two master files. The samtools pipeline was used to identify biallelic single nucleotide polymorphisms (SNPs) relative to the $\mathrm{v} 4$ assembly. The samtools output was filtered to identify SNPs between the resistant and susceptible genotypes and to only include SNPs with a minimum Phred-scaled probability score of QUAL $\geq 25$ and homozygous within a genotype (GT) but unique to each genotype (Additional file 7). Genotype likelihoods ( $\mathrm{PL} \geq 200$ and $\mathrm{PL}=0$; Phred-scaled data likelihoods of possible genotypes) were used to increase the confidence of reported genotypes for reported SNPs. As RNA samples were pooled from multiple resistant or susceptible plants, only SNPs with at least $90 \%$ of a single allele in the resistant and susceptible populations are reported. To predict the relative location of SNPs in the waterhemp genome, we again used the grain amaranth genome, taking advantage of the reciprocal BLASTN $\left(\mathrm{E}<10^{-20},[27]\right)$ described previously.

\section{Results}

Phenotypic assessment of mesotrione responses in resistant and susceptible waterhemp genotypes

Samples used for RNA-seq were harvested prior to the development of visual mesotrione treatment symptoms; therefore, herbicide-treated and mock-treated control plants were maintained in the greenhouse for 3 weeks after mesotrione application to assess phenotype responses. Both genotypes responded to the mesotrione application as expected. The resistant population initially displayed the major HPPD-inhibiting herbicide characteristics of chlorosis and bleached meristematic growth followed by necrosis but recovered by the third week after application. Visual comparison of mock-treated resistant and mock-treated susceptible to mesotrionetreated resistant (Fig. 1) at 3 weeks after treatment showed slight differences, primarily minor stunting and sparse tissue damage within the canopy of the mesotrione-treated resistant population. Conversely, the mesotrione-treated susceptible population sustained heavy tissue bleaching and eventually necrosis and plant death. These observations and comparisons verified the proper herbicide response of both genotypes to mesotrione treatment. 


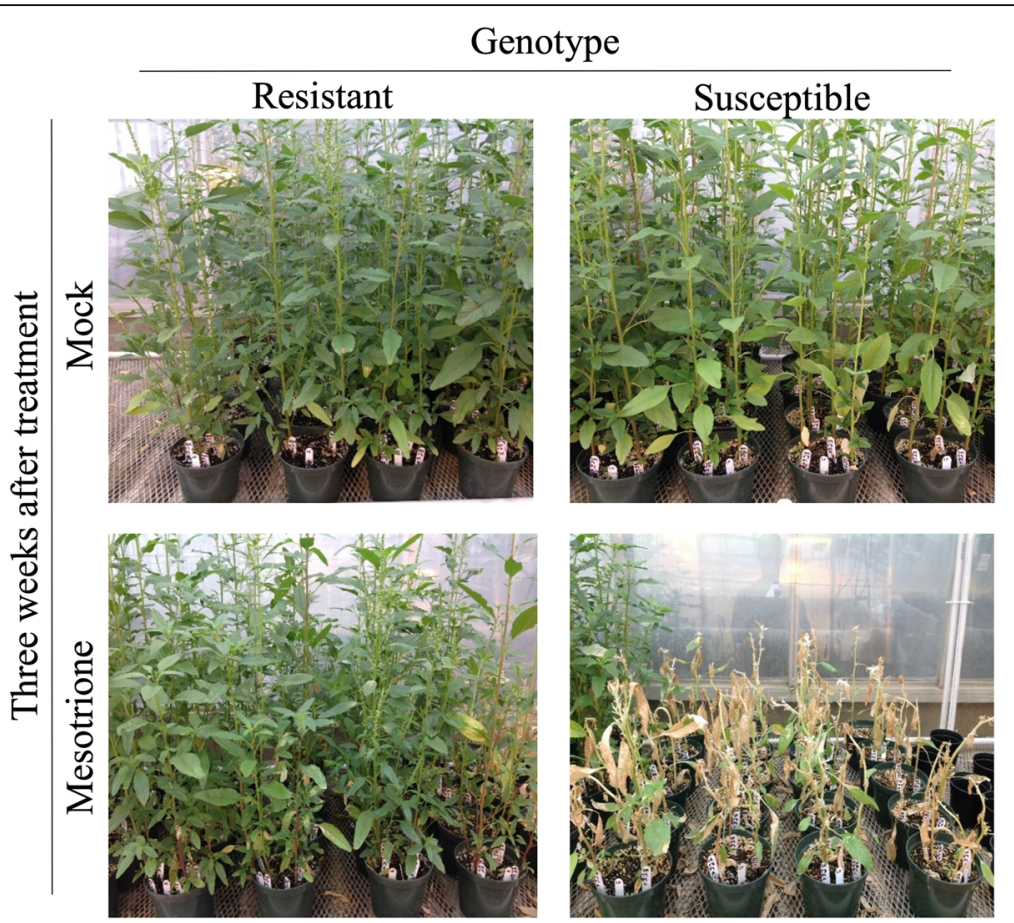

Fig. 1 Phenotyping for mesotrione resistance. To confirm that the waterhemp samples used for RNA-seq analyses were properly treated, an additional set of plants was grown simultaneously with the plants sampled for RNA-seq. No tissues were collected from these plants, instead they were allowed to continue growing for three additional weeks after treatment to assess mesotrione herbicide injury. All plants exhibited the expected phenotype

\section{RNA-Seq and de novo assembly of the waterhemp transcriptome}

Purified RNA from three replicates of 16 samples $(3,6$, 12 , and 24-h samples of mesotrione-treated or mocktreated susceptible and resistant genotypes) were sent to the Iowa State University DNA Facility for the creation and sequencing (100 base pair, paired-end sequencing) of 48 multiplex libraries. A total of 2.45 billion raw reads were produced. Following the sequence clean up described in the Materials and Methods, 2.36 billion sequences were used for de novo transcript assembly using the program Trinity (version 2.0.6, [24]) with three different kmer lengths $(k=25,29$, and 32). Sequences from all samples were used to yield a broad representation of the waterhemp transcriptome and allow the identification of genes expressed in a genotype, treatment or time-specific manner.

When comparing the three assemblies (v1, v2 and v3) generated with differing kmer lengths $(25,29$, and 32 , respectively) we noted that as the kmer length increased, transcript number decreased and Contig N50 increased (Table 1). The Contig N50 is a weighted median of contig (contiguous overlapping sequences) length where $50 \%$ of the assembled nucleotides are contained in contigs greater than or equal to the length of the Contig N50; it can be used as an important measurement in assembly evaluations and was a major factor in the decision of which assembly to use for our analysis [46, 47]. In addition, we visualized contig length distribution for each of our different assemblies (Additional file 1). As suggested by the contig statistics, increasing kmer size increased average contig length and decreased the number of contigs. This was especially evident for contigs smaller than 1000 base pairs $\left(\log _{10} 3\right)$. Therefore, we chose to focus on the third assembly (v3, kmer $=32$ ) for subsequent analysis. Following selection of the v3 assembly, we still needed to remove sequences that lacked open reading frames (ORFs), were redundant, or were expressed at extremely low levels. From the initial v3 assembly containing 451,199 transcripts, TransDecoder [25] was used to identify all transcripts with ORFs> 100 base pairs and remove redundant transcripts, leaving 128,737 transcripts. Similarly, kallisto [26] identified 97,944 lowly-expressed transcripts in the v3 assembly. Cross-referencing the TransDecoder and kallisto datasets resulted in 119,635 transcripts with ORFs $>100 \mathrm{bp}$ and read counts $>10$. Finally, a series of BLASTN analyses described in the materials and methods were used to eliminate transcripts with the best homology to non-plant species or transcripts with no significant hits. This left 113,893 transcripts as the basis of our de novo waterhemp transcriptome (v4) used for differential expression analyses. Sequences for the v4 assembly can be found in Additional file 2. 
Functional annotation of the waterhemp transcriptome The waterhemp v4 assembly was annotated using BLASTX $\left(\mathrm{E}<10^{-10},[27]\right)$ against predicted proteins from $A$. thaliana (The Arabidopsis Information Resource version 10 [TAIR10], [31]), sugar beet (Refbeet v1.2, [28]) and Uniref100 (version 1/22/2016, [32]) and using BLASTN $\left(\mathrm{E}<10^{-20},[27]\right)$ against predicted transcripts from grain amaranth (Phytozome v12.1, Amaranthus hypochondriacus v2.1, [33]). The best $A$. thaliana hits were used to assign the gene ontology (GO) biological processes and the molecular function terms [34] to each transcript of the $\mathrm{v} 4$ assembly. Annotations for the $\mathrm{v} 4$ assembly can be found in Additional file 3 .

To verify the accuracy and coverage of the $\mathrm{v} 4$ assembly, GO biological process terms inferred from homology with $A$. thaliana were mapped to GO slim terms using custom Perl scripts. GO slim term abundance was then compared between the waterhemp v4 transcriptome assembly and all predicted proteins of the A. thali$a n a$, poplar, papaya, asparagus, sugar beet, and grain amaranth genomes (Fig. 2). Waterhemp, sugar beet, and grain amaranth all belong to the Amaranthaceae family $[28,33]$, poplar, papaya and asparagus are dioecious species [35-37], and A. thaliana is a well-established plant model [48]. For each GO slim term, the abundance of assigned transcripts was measured as a percentage relative to the entire transcriptome or genome, allowing us to normalize for any potential genome duplications within a given species. We found that for thirteen of the fourteen GO slim terms, the v4 waterhemp transcriptome assembly was comparable to the genomes of the six other species. This suggests the breadth of the waterhemp v4 transcriptome is consistent with the breadth of the $A$. thaliana, poplar, papaya, asparagus, sugar beet, and grain amaranth genomes. The only exception was the GO slim term 'unknown biological process' which was overrepresented in A. thaliana, compared to the six other species.

\section{Identification of waterhemp transcripts differentially expressed in response to mesotrione}

To allow identification of DETs responding to mesotrione treatment in each genotype, individual samples were mapped to the $\mathrm{v} 4$ waterhemp assembly using the protocol described in the Trinity user manual [24]. In total, $782,456,581$ reads were mapped to the assembly. The raw expression counts were normalized across samples using the Trimmed Mean of M-values (TMM) method [40] in edgeR [41]. Following visual inspection, all replicate samples were considered good quality. Isoforms were considered expressed if they contained at least 1 count per million across three samples or replicates. Of the 113,893 isoforms in the $\mathrm{v} 4$ assembly, 72,697 were considered expressed ( $\mathrm{v} 4$ isoforms). The average length for the expressed isoforms was 1580 base pairs and contigs assumed a normal distribution (Additional file 1).

edgeR was used to identify DETs responding to mesotrione treatment relative to mock-treated controls within each genotype (herbicide resistant and susceptible) across time and at specific time points $(3,6,12$, and $24 \mathrm{~h}$

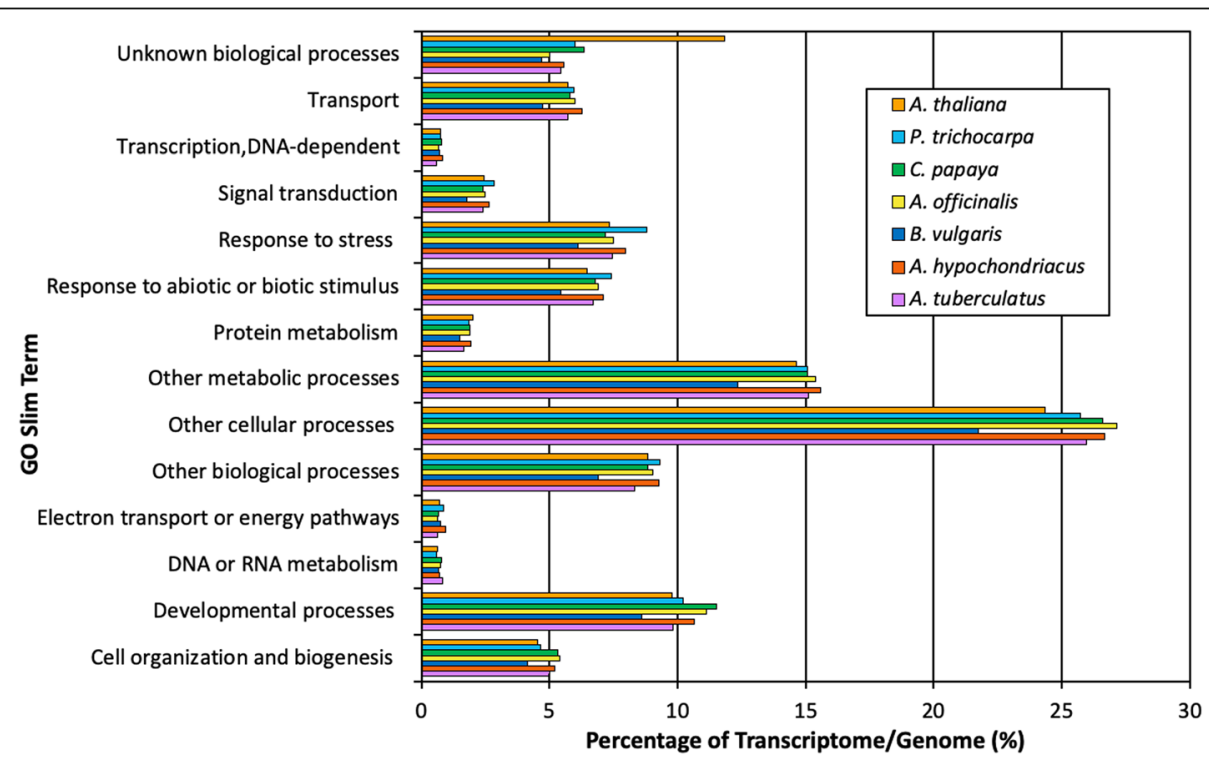

Fig. 2 Comparing the breadth of the waterhemp v4 transcriptome to genomes of related species. Gene ontology biological process (GOBP) slim terms were used to compare the breadth of the waterhemp v4 transcriptome relative to predicted proteins from the Populus trichocarpa, Carica papaya, Asparagus officinalis, Beta vulgaris, Amaranthus hypochondriacus, and Arabidopsis thaliana genomes. The percentage of annotations associated with each GOBP slim term is consistent across all seven species, confirming the waterhemp v4 transcriptome is a suitable proxy to use in the absence of a genome sequence 
after treatment (HAT)). DET expression is reported as a $\log _{2}$ fold change ( $\log _{2}$ FC). A $\log _{2}$ FC greater than 1 indicates a DET is induced by the mesotrione treatment, while a $\log _{2}$ FC less than one indicates a DET is repressed by the mesotrione treatment. DETs with an FDR $<0.05$ [43] are considered significantly differentially expressed in response to mesotrione treatment (Additional file 4).

We identified 89, 62, 61, and 1983 DETs in the resistant waterhemp genotype at $3,6,12$, and 24 HAT, respectively, and 500,77, 61, and 565 DETs were identified in the susceptible waterhemp genotype at $3,6,12$, and 24 HAT, respectively (Table 2). We plotted the number of DETs per genotype within each time point to analyze expression trends across time (Fig. 3). The susceptible waterhemp genotype exhibited large fluxes in DET expression across time. At 3 HAT the susceptible genotype induced 409 transcripts suggesting a quick initial response to the mesotrione treatment. The response diminishes in the middle two time points but then increases again at 24 HAT. In contrast, the resistant waterhemp genotype demonstrated little response to mesotrione treatment at 3, 6 and 12 HAT while a large number of transcripts respond at 24 HAT. Remarkably, while symptoms in response to HPPD herbicide treatments can take as long as 1 week to develop, both resistant and susceptible waterhemp genotypes responded within three HAT. Furthermore, few DETs overlapped between time points within a given genotype (Fig. 4) or between genotypes (Table 2). At 3, 6, 12, and 24 HAT we found $7.7,3.7,8$, and $3.4 \%$ of DETs were common to both waterhemp genotypes, respectively, suggesting a rapid and dynamic response to mesotrione treatment (Table 2).

In addition to identifying transcripts responding to mesotrione treatment at specific time points, we also identified transcripts responding to mesotrione treatment across time. We identified 2091 and 1246 DETs responding to mesotrione treatment across time in the resistant and susceptible genotypes, respectively (Additional file 4). Of these, only 330 DETs were common to both waterhemp genotypes. This reaffirms that the resistant and susceptible genotypes have different responses to the mesotrione treatment.

\section{Characterization of mesotrione responsive transcripts}

While differential expression is useful in identifying individual transcripts found in response to the mesotrione treatment, we were interested in identifying transcripts responding to mesotrione treatment that might have similar functions or act in the same molecular pathway. Therefore, for each time point by genotype combination, we used a Fisher's Exact Test [44] with a Bonferroni correction [45] to identify gene ontology biological process terms [49] significantly overrepresented $(P<0.05)$ among DETs, relative to the waterhemp $v 4$ assembly (Additional file 5). In the resistant waterhemp genotype, we identified 11 and 12 GO terms significantly overrepresented at 3 and 24 HAT. No significant GO terms were identified

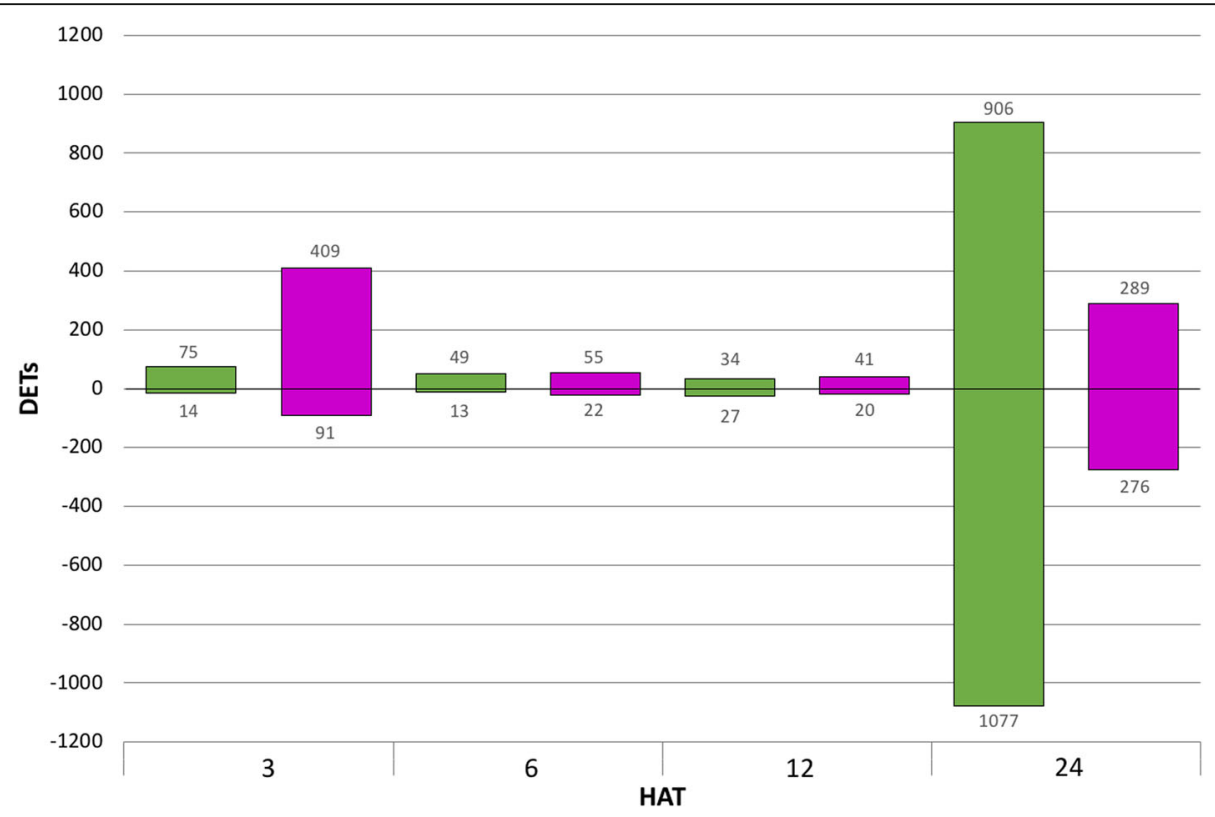

Fig. 3 Identification of waterhemp differentially expressed transcripts (DETs) responding to mesotrione treatment across time. To identify DETs at each time point $(3,6,12$, and $24 \mathrm{~h}$ after treatment (HAT)), transcript expression in resistant (green) or susceptible (magenta) genotypes treated with mesotrione was compared to mock-treated controls. The values above and below the bars represent the number of DETs that were induced and repressed, respectively 


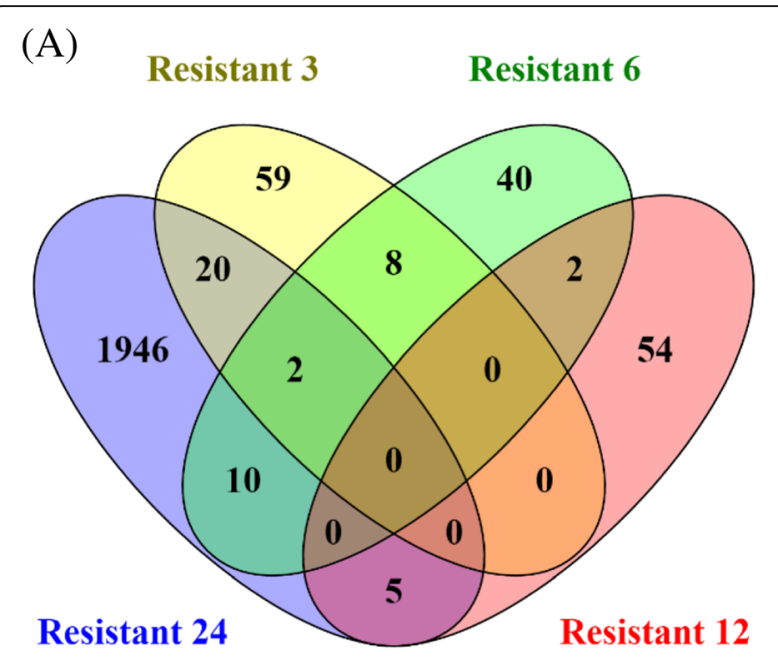

(B)

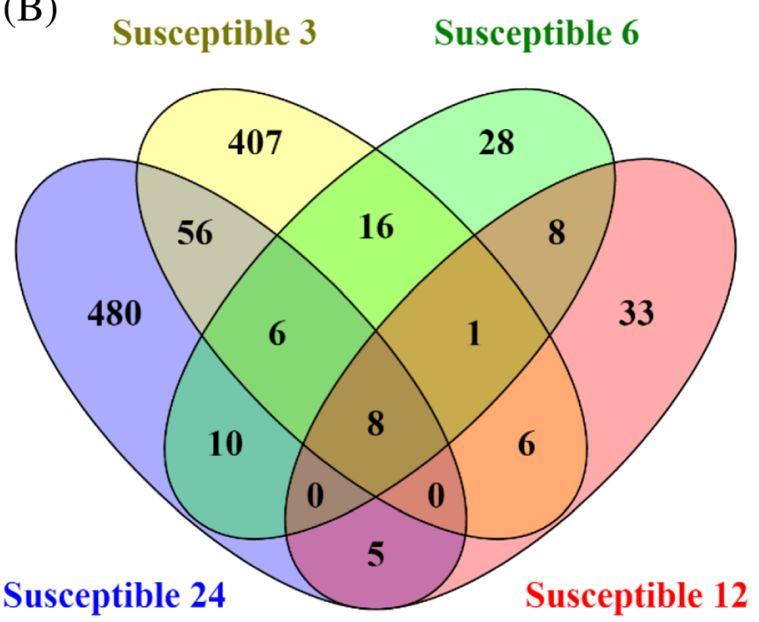

Fig. 4 Mesotrione responsive differentially expressed transcripts (DETs) show little overlap between timepoints. a Comparison of DETs in the resistant genotype at 3, 6, 12, and $24 \mathrm{~h}$ after treatment (HAT). b Comparison of DETs in the susceptible genotype at 3, 6, 12, and $24 \mathrm{HAT}$

at 6 and 12 HAT. Combining all DETs from the resistant waterhemp genotype, we identified 18 significantly overrepresented GO terms. In the susceptible waterhemp genotype, we identified 34, 3, 2 and 24 significant GO terms at 3, 6, 12 and 24 HAT, respectively. Combining all DETs from the susceptible waterhemp genotype, we identified 39 significantly overrepresented GO terms.

To allow direct comparison between resistant and susceptible waterhemp genotypes, we compared unique transcript counts for significant GO terms $(\mathrm{P}<0.05)$ identified at specific time points and over time in both genotypes (Fig. 5). To aid in data visualization, GO terms with DETs that perfectly overlapped with a larger, significant GO term were removed. In addition, only GO terms with at least $10 \mathrm{DETs}$ in either the resistant or susceptible waterhemp genotype are shown. Using this approach, we were able to identify 18 GO terms significantly overrepresented only in the susceptible waterhemp genotype, nine GO terms significantly overrepresented only in the resistant waterhemp genotype and nine GO terms significantly overrepresented in both waterhemp genotypes.

GO terms uniquely overrepresented in the susceptible waterhemp genotype response were largely associated with stress and defense responses including 'response to osmotic stress' (GO:0006970), 'response to hyperosmotic salinity' (GO:0042538), 'response to other organism' (GO:0051707), 'response to virus' (GO:0009615), 'response to wounding' (GO:0009611), and 'respiratory burst involved in defense response' (GO:0002679). Other significantly overrepresented GO terms were associated with metabolism including 'lignin metabolism' (GO:00 09809), 'flavonoid metabolism' (GO:0009813), 'coumarin metabolism' (GO:0009805), 'cellular modified amino acid' (GO:0042398), 'pentacyclic triterpenoid biosynthesis' (GO:0019745), 'sterol biosynthesis' (GO:0016126), 'acetyl-CoA metabolism' (GO:0006084), 'phenylpropanoid metabolism' (GO:0009698), and 'polyamine catabolism' (GO:0006598). Other significant GO terms included 'protein peptidyl-prolyl isomerization' (GO:00 00413), 'peptidyl-proline modification' (GO:0018208), and 'chaperone-mediated protein complex assembly' (GO:0051131). For 11 of the 18 significantly overrepresented GO terms unique to the susceptible waterhemp genotype, we observed more DETs in the susceptible than the resistant genotype.

GO terms significantly overrepresented in both waterhemp genotypes included 'response to cyclopentenone' (GO:0010583), 'response to endoplasmic reticulum stress' (GO:0034976), 'response to hydrogen peroxide' (GO:0042542), 'response to high light intensity' (GO:00 09644), 'response to reactive oxygen species' (GO:00 00302), 'response to cadmium ion stress' (GO:0046686), and 'response to salt stress' (GO:0009651), 'heat acclimation' (GO:0010286), and 'toxin catabolism' (GO:00 09407). For five of the nine GO terms significant in both genotypes, a greater number of DETs were observed in the susceptible waterhemp genotype.

GO terms uniquely overrepresented in the resistant waterhemp genotype were quite varied in their functions. Similar to the responses in the susceptible waterhemp genotype, we identified GO terms associated with response to stress (i.e., 'hyperosmotic response' (GO:0006972), 'response to temperature stimulus' (GO:0009266), and 'response to karrikin' (GO:0080167)). Interestingly, a number of $\mathrm{GO}$ terms were associated with energy metabolism including 'amylopectin biosynthesis' (GO:0010021), 'proteasomal protein catabolism' (GO:0010498), 'gluconeogenesis' (GO:0006094) and 'trehalose biosynthesis' (GO:0005992). Other significant 


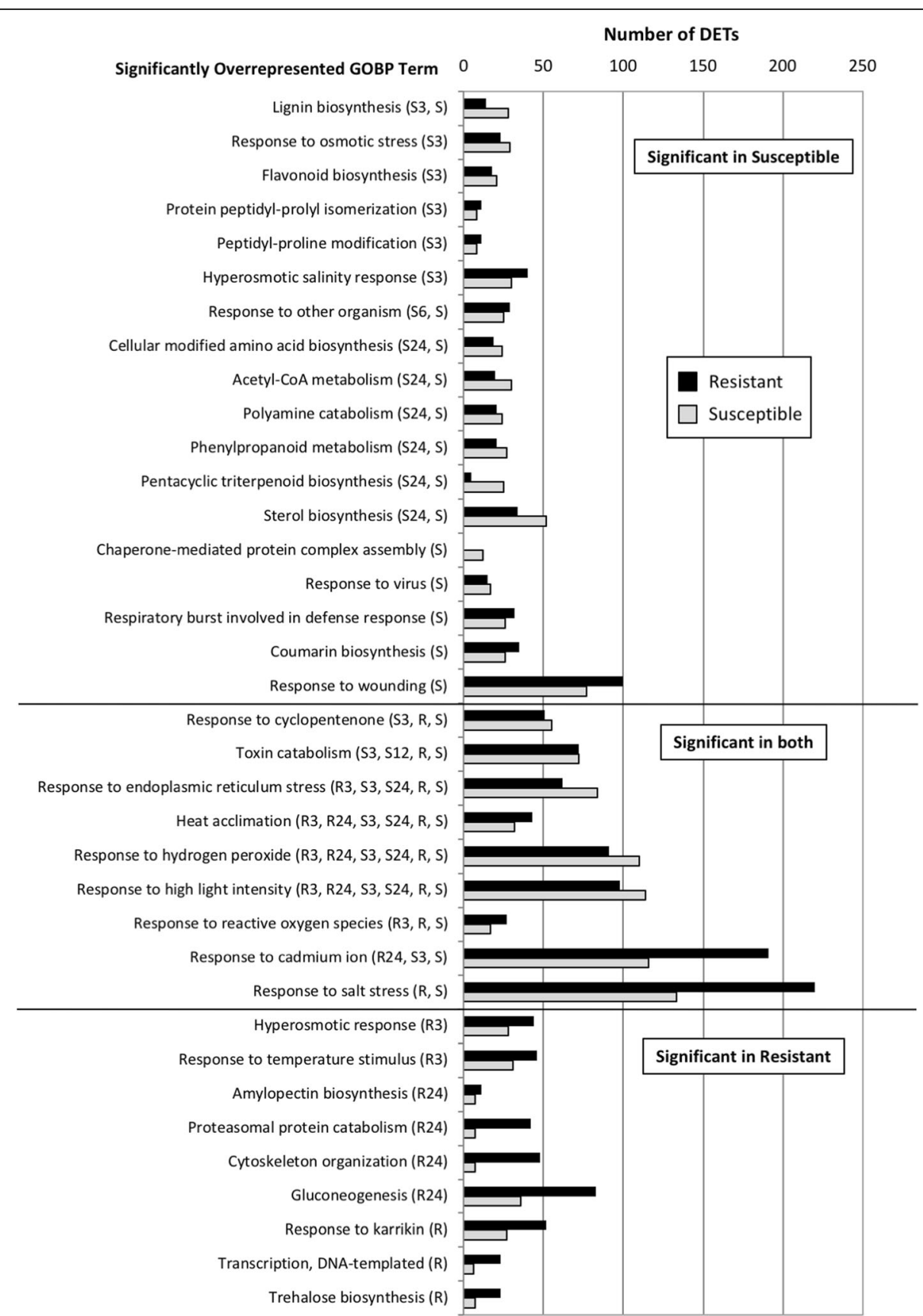

Fig. 5 Characterization of mesotrione responsive differentially expressed transcripts (DETs) using gene ontology (GO) overrepresentation. A Fisher's Exact Test [44] with a Bonferroni correction [45] was used to identify significantly $(P<0.05)$ overrepresented gene ontology biological process terms associated with genotype-specific DETs at a given time point or across time, relative to all transcripts in the waterhemp 44 transcriptome (Additional file 6). For each GO term, all significant time points are indicated in parentheses. To aid in data visualization, GO terms with DETs that perfectly overlapped with a larger, significant GO term were removed. In addition, only GO terms with at least 10 DETs in either the resistant or susceptible genotype are shown. Data is divided to demonstrate significant GO processes unique or common to resistant and susceptible genotypes

GO terms observed in the resistant waterhemp genotype included 'cytoskeleton reorganization' (GO:0007010) and 'transcription' (GO:0006351).

To understand how DETs in these GO terms responded to mesotrione treatment, we compared their expression patterns and expression profiles between resistant and susceptible waterhemp genotypes (Fig. 6). Of the 4799 total DETs, 1311 and 3034 were uniquely significantly differentially expressed in response to mesotrione treatment in the susceptible and resistant 


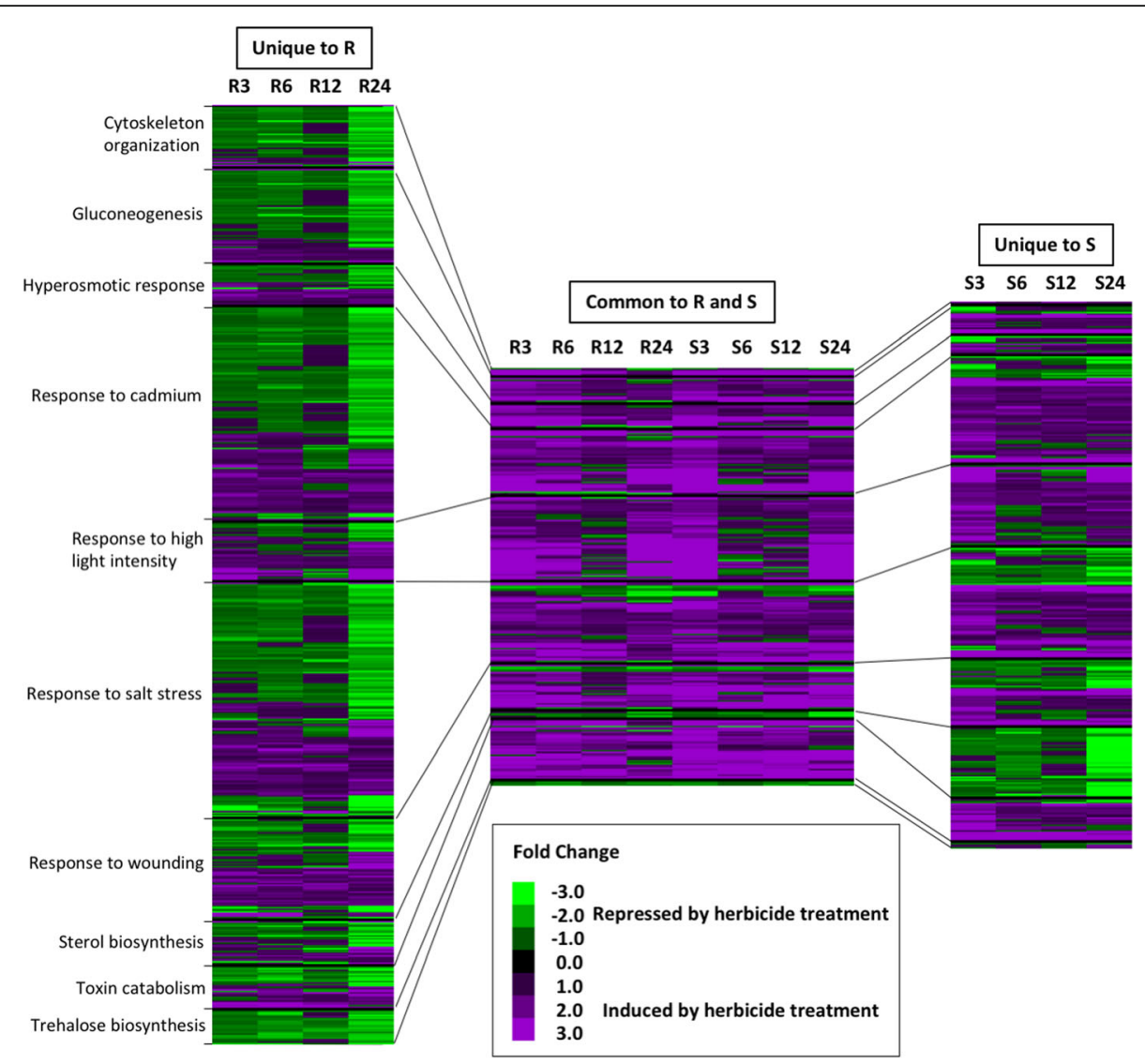

Fig. 6 Characterizing DET expression patterns within a subset of significantly (FDR $<0.05$, [43]) overrepresented GO terms. A core subset of ten gene ontology (GO) terms identified in Fig. 5 were chosen to examine the expression of differentially expressed transcripts (DETs) unique to the resistant (unique to R) or susceptible (unique to S) waterhemp genotypes or common to both (common to R and S). Black lines join portions of the heatmaps for a particular GO term of interest. While DETs common to both genotypes are largely induced, DETs unique to resistant or susceptible genotypes tend to have mixed expression patterns. Further, for some GO terms expression patterns are opposite between unique resistant and unique susceptible DETs. Overall, the expression pattern of induced versus repressed DETs is quite different across time

waterhemp genotypes, respectively. A total of 454 DETs were significantly differentially expressed in both genotypes. We compared DET expression patterns across ten overrepresented gene ontology terms identified above including 'cytoskeleton organization', 'gluconeogenesis', 'hyperosmotic response,' 'response to cadmium,' 'response to high light intensity', 'response to salt stress', 'response to wounding, 'sterol biosynthesis', 'toxin catabolism', and 'trehalose biosynthesis'. When we examined the DETs common to both the resistant and susceptible waterhemp genotypes, we found that the majority of these genes were induced in both genotypes. However, in the susceptible genotype, expression was strongly induced 3 HAT, weakly expressed 6 and 12 HAT, and again strongly induced 24 HAT. A similar response occurred in the resistant genotype, however the dip in gene expression was largely restricted to the 12 HAT timepoint. In contrast, genes repressed in response to mesotrione were weakly repressed at 3,6 , and 12 HAT, but strongly repressed at 24 HAT.
For DETs unique to the susceptible or resistant waterhemp genotypes, we observed differences in the number and expression of DETs depending on the GO terms of interest. The GO terms 'cytoskeleton organization', 'gluconeogenesis', and 'trehalose biosynthesis' were largely unique to the resistant waterhemp genotype response and were repressed by mesotrione treatment. Aside from those DETs common to both genotypes, few DETs were observed in the susceptible waterhemp genotype. For the GO terms, 'response to cadmium,' 'response to salt stress', 'response to high light intensity', and 'toxin catabolism', DETs unique to the susceptible waterhemp genotype were largely induced, while DETs associated with these GO terms in the resistant waterhemp genotype were largely repressed. Unique DETs associated with the GO term 'sterol biosynthesis' were repressed in the susceptible waterhemp genotype but had mixed expression in the resistant waterhemp genotype, while unique DETs associated with the GO terms 'response to wounding' and 
'hyperosmotic response' had mixed expression among unique susceptible and unique resistant DETs.

\section{Clustering of herbicide-responsive DETs}

To identify DETs likely clustered in the waterhemp genome, we used reciprocal BLASTN $\left(\mathrm{E}<10^{-20}\right.$, [27]) to predict transcript location relative to the grain amaranth genome (Phytozome v12.1, Amaranthus hypochondriacus v2.1, [33]) and then tested for overlap using a sliding window of $100,000 \mathrm{bp}$ centered on transcription start sites. We identified 302 unique DETs that fell into 67 clusters (Fig. 7, Additional file 6). Sixty clusters had multiple genotypes, 7 clusters were unique to the resistant genotype, and no cluster was only associated with the susceptible genotype. DET clusters were identified on all grain amaranth chromosomes except for chromosome 16. The most clusters were found on grain amaranth chromosome 6 and the most unique DETs were found on grain amaranth chromosome 12. The biggest cluster of DETs consisted of 8 transcripts. Finding evidence of DET clustering relative to the grain amaranth genome suggests coordinate regulation of gene expression in response to herbicide treatment within clusters.

\section{Identification of candidate single nucleotide polymorphisms}

To identify candidate SNPs that could be used for mapping herbicide resistance and other traits in the future, we called variants between the resistant and susceptible genotypes relative to the $\mathrm{v} 4$ transcriptome. We identified 189 high-quality candidate SNPs (Fig. 8, Additional file 7). Accounting for multiple SNPs located on the same transcript we identified 137 transcripts that contained at least one SNP. To determine if SNP-containing transcripts were associated with specific functions, we queried the biological process GO terms associated with these transcripts. Identified GO terms included: 'nuclear-transcribed mRNA catabolic process' (GO:0000956), 'DNA-templated regulation of transcription' (GO:00 06355), 'protein glycosylation' (GO:0006486), 'response to xenobiotic stimulus' (GO:0009410), 'DNA-templated positive regulation of transcription' (GO:0045893), 'response to ethylene' (GO:0009723), 'response to abscisic acid' (GO:0009737), 'response to gibberellin' (GO:0009739), 'response to salicylic acid' (GO:0009751), 'response to jasmonic acid' (GO:0009753), and 'response to cadmium ion' (GO:0046686). Collectively, these data suggest that SNP-containing transcripts can be associated with defense and stress responses.

To predict the location of SNP-containing transcripts we used reciprocal BLASTN $\left(\mathrm{E}<10^{-20}\right.$, [27] $)$ against transcripts from grain amaranth (Phytozome v12.1, Amaranthus hypochondriacus v2.1, [33]) to identify orthologous genes. SNPs for which a putative ortholog

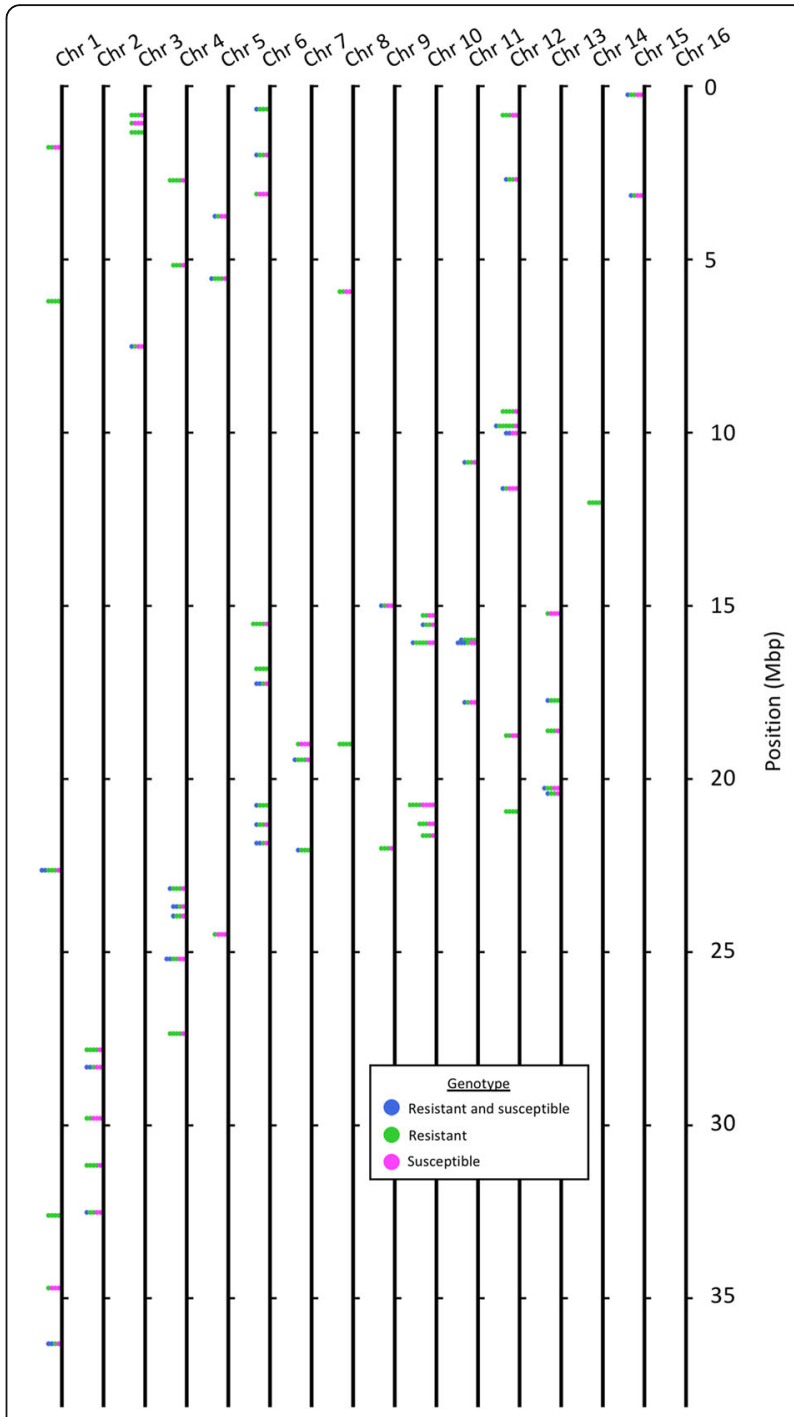

Fig. 7 Clustering of waterhemp DETs based on predicted location relative to Amaranthus hypochondriacus genome. BLASTN $\left(E<10^{-20}\right.$, [27]) was used to predict relative transcript location based on ortholog location in the grain amaranth genome (Phytozome v12.1, Amaranthus hypochondriacus v2.1, [33]). Differentially expressed transcripts (DETs) were tested for overlap in a 100,000 bp window on center ( $\pm 50,000$ bases) from the transcript start site. Neighboring DETs that fell into this window contributed to an average position of the cluster and the number of stacked transcripts. The DETs are color-coded based on the associated genotype(s). Blue circles indicate DETs associated with both the resistant and susceptible genotypes, green circles represent DETs associated with the resistant genotype and magenta circles represent DETs associated with the susceptible genotype. Transcript clusters were identified on all chromosomes except for chromosome 16

could not be confirmed were removed from the mapping analysis. We found candidate SNPs distributed across the grain amaranth genome (Fig. 8, Additional file 7). A high concentration of SNPs (64 of the 118 mapped) was located on chromosome 15. These SNPs correspond to 43 unique transcripts. Several of these transcript 


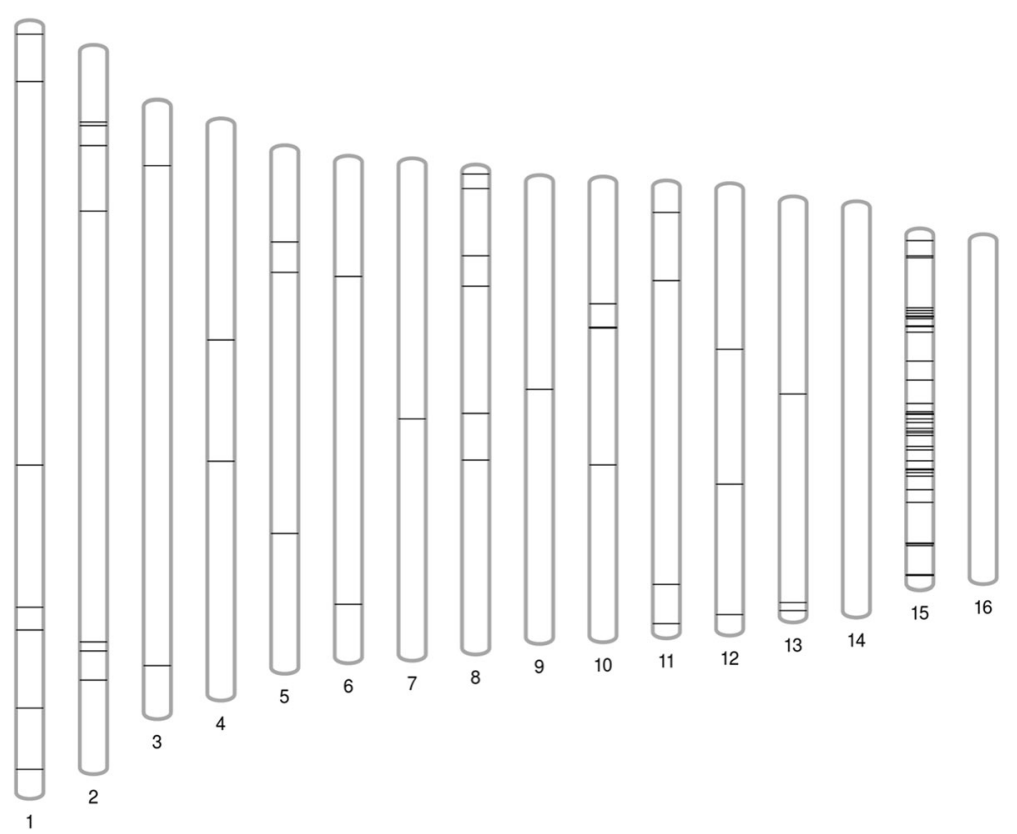

Fig. 8 Mapping of candidate waterhemp single nucleotide polymorphisms (SNPs) relative to the Amaranthus hypochondriacus genome. SNPS were identified by calling SNPs between the resistant and susceptible genotypes relative to the waterhemp v4 transcriptome. BLASTN (E $<10^{-20}$, [27]) was used to predict relative transcript and SNP location based on ortholog location in the grain amaranth genome (Phytozome v12.1, Amaranthus hypochondriacus v2.1, [33]). Of the 189 high-quality SNPs identified, 118 could be reliably mapped to the grain amaranth genome. The highest concentration of SNPs was found on chromosome 15

annotations were associated with defense responses. The SNP concentration and transcript annotations suggest this chromosome could be important for determining herbicide responses in resistant and susceptible populations.

\section{Discussion}

Weeds are the major pest complex in global agricultural systems and will continue to be a problem given the increasing prevalence of herbicide resistance. Soybean and maize fields with high waterhemp population pressure can experience severe yield loss [2,3]. Waterhemp is one of the most prolific and ecologically adapted herbicide resistant weeds found in agricultural fields of the Midwestern United States. Populations with evolved resistance to six different herbicide sites of action including acetolactate synthase (ALS, HG 2, EC 2.2.1.6) inhibitors, synthetic auxins (HG 4), photosystem II (PS II, HG 5, EC 1.10.3.9), 5-enolpyruvyl-shikimate-3-phosphate synthase (EPSPS synthase, HG 9, EC 2.5.1.19), protoporphyrinogen oxidase (PPO, HG 14, EC 1.3.3.4) and 4-hydroxyphenylpyruvate dioxygenase (HPPD, HG 27, EC 1.13.11.27) inhibiting herbicides have been identified [9]. Weediness characteristics associated with waterhemp include rapid growth, self-incompatibility, high seed output and dispersal and ability to compete for space and nutrients with crop species. These characteristics enhance the ability to evolve herbicide resistance and the potential for crop yield loss and subsequent economic impact on farmers and agricultural production. Thus, waterhemp is an important species to study by agricultural and plant science communities.

Several Amaranthus species, including waterhemp, have been discussed as potential candidates for genomic efforts $[50,51]$. Supporting the waterhemp initiative was the identification of populations with five-way resistance to herbicides with different sites of action in Iowa in 2011 [52]. In this study, we used the mesotrione resistant population characterized by Kaundun et al. [15]. Mesotrione resistance in this population was not due to mutation, amplification, or increased expression of the herbicide target gene HPPD. Rather, biochemical analyses suggest cytochrome P450s contribute to increased herbicide metabolism. Significant differences in herbicide metabolism were not detected until $48 \mathrm{~h}$ after treatment. This suggests that herbicide responses are inducible and genes upstream of the cytochrome P450s directly or indirectly respond to herbicide treatment. To identify these early-acting genes and gene networks, we needed to establish genomic technologies for waterhemp. These tools are critically important for global food security [53].

\section{Development of genomic resources for studying traits of interest in waterhemp}

Initial genomic studies of waterhemp using 454 pyrosequencing were able to characterize several herbicide 
resistance target genes [20, 21]. However, these studies pooled RNA samples of different tissues and treatments prior to sequencing, making it impossible to directly differentiate herbicide treatment responses in resistant and susceptible waterhemp genotypes. In contrast, our sequencing and de novo transcriptome assembly approach used 48 multiplexed libraries representing resistant and susceptible waterhemp genotypes, treated and mocktreated with the HPPD herbicide mesotrione across a twenty-four hour time course. Assembling RNA-seq data across libraries allowed us to develop a comprehensive waterhemp leaf transcriptome, which represents an important asset to various scientific communities. Comparisons of expressed genes in crop species verses weeds could provide insights into novel targets for weed specific genes for future weed control. Ecologists, plant and weed scientists could utilize this tool to study important weed traits or provide information on leaf development and photosynthesis that might be applied to crop improvement.

Assembly statistics of our waterhemp transcriptome (Table 1, Additional file 2) coupled with comparisons to predicted proteins from the model species A thaliana, and the related species sugar beet and grain amaranth (Fig. 2) confirm the quality and breadth of our assembly. To increase the utility of the waterhemp transcriptome, the Additional data files include assembled sequences for the v4 assembly and a database of annotated transcripts. Furthermore, raw sequences have been deposited in the National Center for Biotechnology Small Reads Archive (NCBI SRA, Bioproject PRJNA432348 and SRA Study SRP132642) allowing for reassembly and continued improvement as more sequences from waterhemp populations become available.

To demonstrate how these data can be used by the weed science community, we mined the waterhemp transcriptome (Additional file 3) for any transcript containing 'CYP' or 'P450' in its annotation (Table 3). Recently, Kaundun et al. [15] attributed HPPD-resistance in the Tarnov, Nebraska population to increased herbicide metabolism through the activity of cytochrome P450s. We identified 970 putative cytochrome P450s. Similar mining of the DET file (Additional file 4) identified 79 herbicide responsive DETs with homology to cytochrome P450s. Of these 29 were significantly differentially expressed in the resistant population, 34 were significantly differentially expressed in the susceptible population and 16 were differentially expressed in both. Of great interest was transcript TR102135, which was the only transcript significantly induced in the resistant genotype at 3 hours. Ten cytochrome P450 transcripts were identified only in the resistant genotype at 24 HAT. Kaundun et al. [15], found the mesotrione metabolite 4-hydroxymesotrione began to accumulate in the resistant parent between 24 and $48 \mathrm{~h}$ after herbicide treatment. However, we detected differential expression of cytochrome P450s in the resistant population well before this time. This suggests genes upstream of cytochrome P450s can recognize and respond to herbicide treatment.

Of the 79 herbicide-responsive transcripts with homology to cytochrome P450s, three were clustered relative to the grain amaranth genome (cluster 21, TR87604, TR34266 and TR3711, Additional file 6). We also identified two SNP-containing transcripts (TR33255 and TR9764) annotated as cytochrome P450s from our SNP data (Additional file 7). TR33255 was homologous to the Arabidopsis gene, AT2G29090.2, which encodes a cytochrome P450 (CYP707A2) with abscisic acid (ABA) 8 -hydroxylase activity $[31,54]$. This protein belongs to the CYP707A gene family and is associated with controlling ABA levels in late seed maturation through germination [55]. ABA is a phytohormone involved in plant response to abiotic stress and has also been associated with protecting plants from herbicide damage $[56,57]$. Devine et al. [57] demonstrated that applying exogenous ABA helped protect oats (Avena sativa L.) against applications of diclofop-methyl or low rates of tralkoxydim. While these transcripts did not respond to herbicide

Table 3 Summary of candidate DETs for non-target site herbicide resistance and circadian rhythm associated proteins

\begin{tabular}{llll}
\hline & $\begin{array}{l}\text { Keywords used } \\
\text { in file search }\end{array}$ & Number of Hits & Differentially Expressed \\
\cline { 2 - 3 } Cytochrome P450 & $\begin{array}{l}\text { "CYP" } \\
\text { "P450" }\end{array}$ & 79 \\
Glutathione Stransferase & "GST" & 970 & 47 \\
"Glutathione Stransferase" & 157 & 69 \\
"glycosyltransferase" & "glucosyltransferase" & 689 & 35 \\
protein & "ABC transporter" & 644 & 35 \\
Circadian rhythm & "ABC protein" & 833 & 42 \\
\hline
\end{tabular}

The waterhemp transcriptome (Additional file 3) and significantly differentially expressed transcripts (Additional file 4) were mined for non-target site herbicide resistance and circadian rhythm associated transcripts using combinations of keywords to identify putative candidate transcripts for further investigation 
treatment in our analyses, they could represent non-inducible cytochrome P450s that could be differentially expressed between the resistant and susceptible genotypes or may be differentially expressed at timepoints not evaluated in this study. Similar approaches could be used to mine the data for sites-of-action for other important herbicides, non-target site herbicide resistance associated genes, or for traits of interest.

\section{Identification of transcripts responding to mesotrione treatment in waterhemp}

Mapping of reads from specific waterhemp genotypes, mesotrione and mock treatments and time points allowed us to leverage our waterhemp transcriptome to identify the genes and gene networks responding to mesotrione treatment in waterhemp. A general trend we observed was that the number of DETs was greatest at 3 and 24 HAT (Fig. 3) in both the resistant and susceptible genotype, suggesting a biphasic response to herbicide treatment. In both the resistant and susceptible waterhemp genotypes, mesotrione treatment resulted in the differential expression of transcripts associated with stress and defense responses. When we compared gene expression patterns in genes common to the resistant and susceptible interaction (Fig. 6), we again see a biphasic response. Given that DETs in both waterhemp genotypes at 3 and 24 HAT were significantly overrepresented with GO terms associated with light (i.e., 'response to high light intensity', 'response to red light', and 'response to far red light'), it's possible that circadian rhythm could influence differential gene expression in response to herbicide treatment. However, the 3, 6 and 24 HAT timepoints were collected during daylight, so it seemed unlikely circadian rhythm was involved. To confirm this, we mined the DETs in each genotype by timepoint combination for the term 'circadian' (Table 3). We identified 28 and 17 herbicide responsive DETs with annotations associated with circadian rhythm in the resistant and susceptible genotype, respectively. In the resistant genotype, 0, 0, 0, and 15 circadian clock associated DETs were expressed specifically at R3, R6, R12, and R24. In the susceptible genotype, we identified 8, 1 , 1 , and 5 circadian clock associated DETs at S3, S6, S12, and S24, respectively. This suggests herbicide treatment could impact circadian rhythm.

Biphasic gene expression patterns have been observed in response to biotic (reviewed by [58]) and abiotic $[59,60]$ stress and in response to mechanical wounding [61]. Reactive oxygen species (ROS) have now been tied to biphasic gene expression in defense, response to wounding, high light conditions, and abiotic stress (reviewed by [62]). HPPD-inhibitor herbicides disrupt photosynthesis and the production of carotenoids that protect plants from UV damage leading to the accumulation of ROS [63]. The GO terms 'response to hydrogen peroxide' (FDR $<0.05, \mathrm{R}, \mathrm{R} 3, \mathrm{R} 24$, $\mathrm{S}, \mathrm{S} 3$, and S24) and 'response to reactive oxygen species' (FDR $<0.05, \mathrm{R} 3, \mathrm{R}$, and $\mathrm{S}$ ) were both significantly overrepresented in resistant and susceptible genotypes, suggesting that herbicide treatment can also induce biphasic responses in weeds, likely through the action of ROS.

When we compared DET expression in transcripts unique to the resistant or susceptible waterhemp genotypes, we observed that DETs associated with 'response to cadmium', 'response to high light', 'hyperosmotic response', 'response to salt stress', and 'toxin catabolism' were largely induced by mesotrione treatment in the susceptible genotype across the time course. In contrast, DETs unique to the resistant waterhemp genotype and associated with these same GO terms were largely repressed across time (Fig. 6). These data highlight several remarkable features of the resistant waterhemp genotype's response to mesotrione. First, responses to mesotrione treatment were detected very quickly, within three HAT in both resistant and susceptible waterhemp genotypes. Second, while the susceptible waterhemp genotype continued to induce stress responses over the experiment time course, by three HAT the resistant waterhemp genotype was already repressing differential expression of stress-associated genes. This suggested that by three HAT, the resistant waterhemp genotype began to neutralize herbicidal activity and was likely returning to normal physiological function.

Using the reference genome of grain amaranth, we were able to identify groups of DETs that clustered together by predicted genomic locations. The clustered DETs were generally associated with detoxification and stress responses. Toxin metabolism in plants occurs in three phases: transformation, conjugation and compartmentation. The two major enzymes associated with phase I and phase II of detoxification (cytochrome P450 monooxygenase and glutathione transferase, respectively) were among the clustered DETs [64]. Among the annotations for the clustered DETs we found multiple enzymes associated with all three phases of pesticide and xenobiotic metabolism such as alcohol or aldehyde dehydrogenase, glycosyltransferase, methyltransferase, and ATP-binding cassette (ABC) transporters [65] (Table 3). Other DETs have associations with stress response and signaling such as heat shock proteins and lipoxygenase $[66,67]$. These associations of clustered DETs with xenobiotic detoxification and stress signaling support potential coordinate regulation of defense response genes by waterhemp in response to mesotrione treatment.

We cross-referenced the 189 SNP-containing transcripts with the total 4799 herbicide responsive DETs. We identified 18 unique herbicide responsive transcripts 
that contained SNPs. Four transcripts with documented herbicide response were easily identifiable. TR45906|c2_g1_i2 was homologous to the Arabidopsis gene CYP73A5, a member of the cytochrome P450 enzyme family [68]. TR16523|c2_g1_i4 and TR36394|c2_g7_i1 were homologous to the UDP-glucosyl transferase, UGT73B3. Lim et al. [69] found UGT73B3 recessive mutants resistant to paraquat, a photosystem I (PSI, HG 22, EC 1.97.1.12) electron diverting herbicide. TR18103|c1_g2_i1 was homologous to UGT74E2, which was also reported in the differential response of Arabidopsis to ALS-inhibiting (HG 2) and EPSPS-inhibiting (HG 9) herbicides [70]. Given that these SNPs correspond to herbicide responsive DETs, they are high priority candidates for future marker development.

While responses to light and stress were expected, our analyses determined that DETs unique to the resistant waterhemp genotype were significantly overrepresented with the GO terms 'cytoskeleton organization', 'gluconeogenesis', and 'trehalose biosynthesis'. Genes within these
GO terms were significantly repressed in response to the mesotrione treatment, especially at 24 HAT. To connect these responses and examine the underlying gene networks, we took advantage of the waterhemp annotation platform to identify the best $A$. thaliana homologs for DETs associated with these GO terms. Unique $A$. thaliana identifiers were then submitted to the STRING website to identify gene networks [71]. Networks in STRING are established using a variety of methods including but not limited to experiments, public databases and co-expression. Collectively, the three GO terms contained 91 DETs which corresponded to 45 unique $A$. thaliana identifiers. Of these, 36 could be assigned to the same network with a confidence score ranging from 0.41 to 0.99 (Fig. 9). Interestingly, the network also contained several genes associated with herbicide resistance. This included two beta-tubulins inhibitors (AtTUB6 and AtTUB8), a glutamyl-tRNA synthetase (At5g26710), an ascorbate peroxidase $(A P X 1)$, a superoxide dismutase

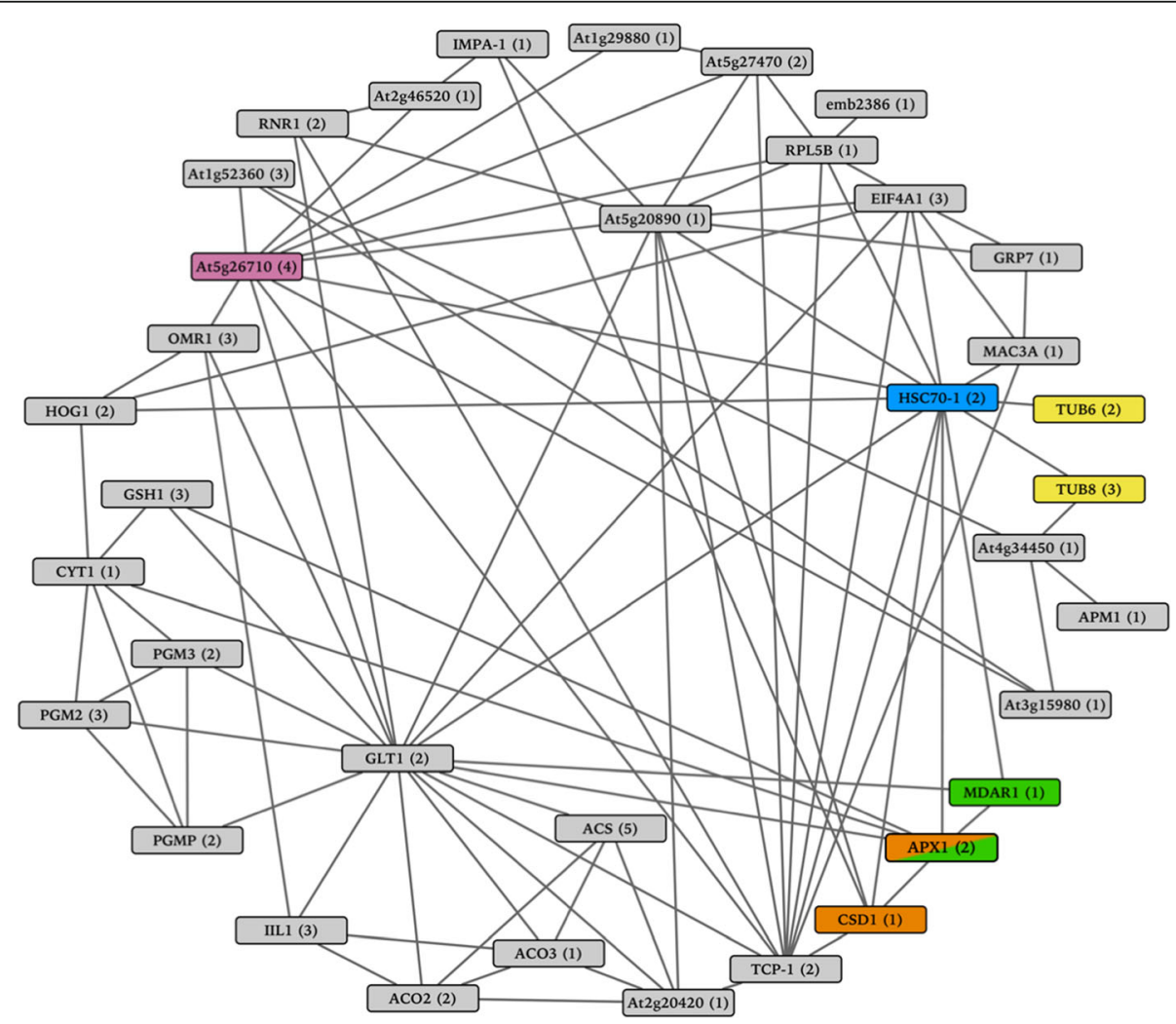

Fig. 9 Identification of gene networks unique to mesotrione resistance associated with the GO terms gluconeogenesis, cytoskeleton organization and trehalose biosynthesis. We took advantage of the waterhemp (Amaranthus tuberculatus) annotation platform to identify the best Arabidopsis thaliana homologs for 91 unique resistant DETs associated with these GO terms. Forty-five unique $A$. thaliana identifiers were then submitted to the STRING website to identify potential gene networks [71]. All 45 unique A. thaliana identifiers were present in the database and 36 could be combined in a single network. Lines linking proteins indicate connections between proteins. The numbers in parentheses indicate the number of unique resistant DETs that correspond to each Arabidopsis gene ID. The yellow nodes highlight genes that have associations with dinitroaniline ( $H G$ 3) herbicide resistance, the purple node highlights an association with 2,4-D (HG 4) herbicide resistance, the orange nodes highlight associations with atrazine ( $H G$ 5) herbicide resistance and the green nodes highlight associations with paraquat (HG 22) herbicide resistance. The blue node highlights the only node in this network that interacted with all six nodes associated with herbicide resistance 
(CSD1) and a monodehydroascorbate reductase (MDAR1). Anthony et al. [72] found that co-overexpression of mutant alpha and beta tubulins generated dinitroaniline (HG 3) herbicide-resistant tobacco (Nicotiana tabacum L.). While studying gene expression patterns of naturally tolerant wheat (Triticum aestivum L., variety Greina), Pasquer et al. [73] found glutamyl-tRNA synthetase was significantly induced by $2,4-\mathrm{D}$ (2,4-dichlorophenoxyacetic acid, HG 4). Jiang et al. [74] found increased expression of superoxide dismutase and ascorbate peroxidase in response to atrazine (HG 5) treatment in pearl millet (Pennisetum americanum (L.) K. Schum). Murgia et al. [75] found that overexpression of ascorbate peroxidase in Arabidopsis also conferred resistance to paraquat (HG 22). Monodehydroascorbate reductase activity was correlated to paraquat (HG 22) resistance in horseweed (Conyza canadensis (L.) Cronquist) [76]. Interestingly, all six gene nodes interacted with the heat shock $70 \mathrm{kDa}$ protein (HSC70-1) node in our network (Fig. 9). Additionally, two genes were identified that had associations with herbicide resistance but did not interact with the larger gene network, a trehalose-6-phosphate phosphatase (TPPD) and a regulatory particle AAA-ATPase 2A (RPT2a) [77, 78]. These findings, mirroring the expression we observe in the waterhemp DETs, suggest that many herbicides thought to have unique targets may actually be acting on the same gene networks.

\section{Conclusions}

Many important plant species to agriculture lack well-curated reference genomes. However, RNA-seq has allowed scientific communities to develop transcriptomes for characterizing genes and traits important for agronomic performance of both crops and weeds. In this study, we provide a comprehensive resource for waterhemp genomics, including the first waterhemp transcriptome, identification of DETs responding to herbicide treatment in HPPD-resistant and susceptible genotypes and candidate SNPs for future marker development. While other studies have sequenced the waterhemp transcriptome and identified important herbicide targetsites, this is the first transcriptomic analysis that identifies genes and gene networks that are differentially expressed in response to HPPD inhibiting herbicides in HPPD-resistant and susceptible waterhemp. Our analyses reveal 1) that waterhemp responses to mesotrione are quick and detectable in as little as 3 hours, 2) differential gene expression in resistant and susceptible waterhemp genotypes show little overlap in mesotrione responses, 3) genes targeted by other herbicides may belong to the same gene networks providing insight into the evolution of herbicide resistance and 4) predicted locations of DETs suggest coordinate regulation of defense responses in HPPD-resistant waterhemp. These findings lay a strong foundation for future research and will improve opportunities to better understand and manage weeds with evolved resistances to herbicides.

\section{Additional files}

Additional file 1: Contig length distribution of the four versions of the de novo waterhemp (Amaranthus tuberculatus) transcriptome assemblies. Assemblies $v 1, v 2$ and $v 3$ were generated with differing kmer lengths ( 25 29 , and 32 , respectively) as described in the materials and methods (PNG $175 \mathrm{~kb}$ )

Additional file 2: Waterhemp (Amaranthus tuberculatus) v4 transcriptome sequences in FASTA format. (XLSX $21386 \mathrm{~kb}$ )

Additional file 3: Annotation of the waterhemp (Amaranthus tuberculatus) version 4 transcriptome. BLASTX [26] was used to compare waterhemp transcriptome sequences against the Uniref100 (version 01/ 22/2016, [31]) nonredundant protein database. (XLSX 919 kb)

Additional file 4: dentification of waterhemp (Amaranthus tuberculatus) transcripts significantly (FDR $<0.05,[43])$ differentially expressed in response to mesotrione treatment. edgeR was used to identify differentially expressed transcripts (DETs) responding to herbicide treatment relative to mock-treated controls within each genotype (herbicide resistant (R) and susceptible(S)) and at specific time points $(3,6,12$, and $24 \mathrm{~h}$ after treatment (HAT). (XLSX $18 \mathrm{~kb}$ )

Additional file 5: Characterization of waterhemp (Amaranthus tuberculatus) differentially expressed transcripts (DETs) using gene ontology overrepresentation. A Fisher's Exact Test [42] with a Bonferroni correction [43] was used to identify significantly $(P<0.05)$ overrepresented gene ontology biological process terms among DETs significant within a genotype at a particular time or across time, relative to all transcripts in the waterhemp v4 transcriptome. (XLSX $141 \mathrm{~kb}$ )

Additional file 6: Waterhemp (Amaranthus tuberculatus) differentially expressed transcripts (DETs) cluster relative to Amaranthus hypochondriacus genome. BLASTN $\left(E<10^{-20}\right.$, [26]) was used to predict relative transcript location based on ortholog location in the grain amaranth genome (Phytozome v12.1, Amaranthus hypochondriacus v2.1, (XLSX 73 kb)

Additional file 7: Mapped candidate single nucleotide polymorphisms (SNPs) identified between mesotrione-resistant and susceptible waterhemp (Amaranthus tuberculatus (GZ 19875 kb)

\section{Abbreviations}

ABA: abscisic acid; ABC: ATP-binding cassette; ACC: aminocyclopropane-1carboxylic acid; ALS: aceltolactate synthase; COC: crop oil concentrate; contig: contiguous overlapping sequences; CPM: count per million; CYP: cytochrome P450; DETs: differentially expressed transcripts; EPSPS: 5enolpyruvyl-shikimate-3-phosphate; FDR: false discovery rate; GFF: general feature format; GO: gene ontology; GOBP: gene ontology biological process; HAT: hours after treatment; HG: herbicide group; HGA: 2,5dihydroxyphenylacetate; HPP: 4-hydroxyphenylpyruvate; HPPD: 4hydroxyphenylpyruvate-dioxygenase; $\log _{2}$ FC: $\log _{2}$ fold change; NCBI SRA: National Center for Biotechnology Small Reads Archive; ORF: open reading frame; PPO: protoporphyrinogen oxidase; PS: photosystem; RNAseq: RNA-sequencing; ROS: reactive oxygen species; RSEM: RNA-seq by expectation-maximization;: SNP: single nucleotide polymorphism; TAIR10: The Arabidopsis Information Resource version 10; TMM: trimmed mean of Mvalues; UAN: urea ammonium nitrate

\section{Acknowledgements}

Appreciation is extended to Eric A.L. Jones for helpful discussion, Hamze Dokoohaki for valuable assistance in the visualization of DET data relative to the grain amaranth genome and Ms. Lori Lincoln for technical assistance. The USDA is an equal opportunity provider and employer. Mention of trade names or commercial products in this article is solely for the purpose of 
providing specific information and does not imply recommendation or endorsement by the U. S. Department of Agriculture.

\section{Funding}

This research was financed in part by funds provided by the United States Department of Agriculture, Agricultural Research Service (USDA-ARS) CRIS Project 3625-21220-005-00D, the lowa Soybean Association (ISA), and the lowa State University Department of Agronomy. None of the funding bodies were involved with the research other than providing funds to support the work

\section{Availability of data and materials}

The raw sequences generated during the current study are available in the National Center for Biotechnology Small Reads Archive (NCBI SRA, http:// www.ncbi.nlm.nih.gov/sra) under Bioproject PRJNA432348 and SRA Study SRP132642. The datasets generated or analyzed during this study are included in this published article and its additional files.

\section{Authors' contributions}

DRK, JAO, MDKO and MAG designed the experiment. DRK, JAO and MAG collected data and performed data analyses. DRK, JAO, MDKO and MAG prepared the manuscript. All authors read and approved the final manuscript.

\section{Ethics approval and consent to participate}

Collections of plant material comply with institutional, national, and international guidelines.

\section{Consent for publication}

Not applicable.

\section{Competing interests}

The authors declare that they have no competing interests.

\section{Publisher's Note}

Springer Nature remains neutral with regard to jurisdictional claims in published maps and institutional affiliations.

Received: 18 January 2019 Accepted: 22 April 2019

Published online: 06 May 2019

\section{References}

1. Steckel LE. The dioecious Amaranthus spp.: here to stay. Weed Technol. 2007:21:567-70

2. Steckel LE, Sprague CL. Common waterhemp (Amaranthus rudis) interference in soybean. Weed Sci. 2002:52:359-64.

3. Bensch CN, Horak MJ, Peterson D. Interference of redroot pigweed (Amaranthus retroflexus), palmer amaranth (A. palmeri), and common waterhemp (A. rudis) in soybean. Weed Sci. 2003;51:37-43.

4. Steckel LE, Sprague CL, Hager AG, Simmons FW, Bollero GA. Effects of shading on common waterhemp (Amaranthus rudis) growth and development. Weed Sci. 2003;51:898-903.

5. Hartzler RG, Buhler DD, Stoltenberg DE. Emergence characteristics of four annual weed species. Weed Sci. 1999:47:578-84

6. Horak MJ, Loughin TM. Growth analysis of four Amaranthus species. Weed Sci. 2000;48:347-55.

7. Powles SB, Preston C, Bryan IB, Jutsum AR. Herbicide resistance: impact and management. Adv Agron. 1997;58:57-93.

8. Ryan GF. Resistance of common groundsel to simazine and atrazine. Weed Sci. 1970:18:614-6.

9. Heap I. The International Survey of Herbicide Resistant Weeds [Internet] [cited 2019 February 19]. Available from: http://www.weedscience.org

10. Hausman NE, Singh S, Tranel PJ, Riechers DE, Kaundun SS, Polge ND, et al Resistance to HPPD-inhibiting herbicides in a population of waterhemp (Amaranthus tuberculatus) from Illinois, United States. Pest Manag Sci. 2011 67:258-61.

11. McMullan PM, Green JM. Identification of a tall waterhemp (Amaranthus tuberculatus) biotype resistant to HPPD-inhibiting herbicides, atrazine, and thifensulfuron in lowa. Weed Technol. 2011;25:514-8.
12. Matringe $M$, Sailland A, Pelissier B, Rolland A, Zink O. p Hydroxyphenylpyruvate dioxygenase inhibitor-resistant plants. Pest Manag Sci. 2005;61:269-76.

13. Ma R, Kaundun SS, Tranel PJ, Riggins CW, McGinness DL, Hager AG, et al Distinct detoxification mechanisms confer resistance to mesotrione and atrazine in a population of waterhemp. Plant Physiol. 2013;163:363-77.

14. Oliveira MC, Jhala AJ, Gaines T, Irmak S, Amundsen K, Scott JE, et al. Confirmation and control of HPPD-inhibiting herbicide-resistant waterhemp (Amaranthus tuberculatus) in Nebraska. Weed Technol. 2017:31:67-79.

15. Kaundun SS, Hutchings SJ, Dale RP, Howell A, Morris JA, Kramer VC, et al. Mechanism of resistance to mesotrione in an Amaranthus tuberculatus population from Nebraska, USA. PLoS One. 2017;12:1-22.

16. Délye $C$, Jasieniuk $M$, Le Corre $V$. Deciphering the evolution of herbicide resistance in weeds. Trends Genet. 2013;29:649-58.

17. Huffman J, Hausman NE, Hager AG, Riechers DE, Tranel PJ. Genetics and inheritance of nontarget-site resistances to atrazine and mesotrione in a waterhemp (Amaranthus tuberculatus) population from Illinois. Weed Sci. 2015;63:799-809.

18. Oliveira MC, Gaines TA, Jhala AJ, Knezevic SZ. Inheritance of mesotrione resistance in an Amaranthus tuberculatus (var. rudis) population from Nebraska, USA. Front Plant Sci. 2018:9:1-12.

19. Kohlhase DR, Edwards JW, Owen MDK. Inheritance of 4-hydroxyphenylpyruvate dioxygenase inhibitor herbicide resistance in an Amaranthus tuberculatus population from lowa, USA. Plant Sci. 2018;274:360-8.

20. Lee RM, Thimmapuram J, Thinglum KA, Gong G, Hernandez AG, Wright CL, et al. Sampling the waterhemp (Amaranthus tuberculatus) genome using pyrosequencing technology. Weed Sci. 2009;57:463-9.

21. Riggins CW, Peng Y, Stewart CN, Tranel PJ. Characterization of de novo transcriptome for waterhemp (Amaranthus tuberculatus) using GS-FLX 454 pyrosequencing and its application for studies of herbicide target-site genes. Pest Manag Sci. 2010;66:1042-52.

22. Buffalo V. Scythe - a Bayesian adapter trimmer [internet]. UC Davis Bioinforma. Core. Available from: https://github.com/ucdavis-bioinformatics/scythe

23. Joshi N, Fass J. Sickle: a sliding-window, adaptive, quality-based trimming tool for FastQ files (version 1.33) [internet]. UC Davis Bioinforma Core Available from: https://github.com/ucdavis-bioinformatics/sickle

24. Grabherr MG, Haas BJ, Yassour M, Levin JZ, Thompson DA, Amit I, et al. Fulllength transcriptome assembly from RNA-Seq data without a reference genome. Nat Biotechnol. 2011;29:644-52.

25. Haas BJ, Papanicolaou A, Yassour M, Grabherr M, Blood PD, Bowden J, et al. De novo transcript sequence reconstruction from RNA-seq using the trinity platform for reference generation and analysis. Nat Protoc. 2013;8:1494-512.

26. Bray NL, Pimentel H, Melsted P, Pachter L. Near-optimal probabilistic RNASeq quantification. Nat Biotechnol. 2016;34:525-7.

27. Altschul SF, Madden TL, Schäffer AA, Zhang J, Zhang Z, Miller W, et al. Gapped BLAST and PS I-BLAST: a new generation of protein database search programs. Nucleic Acids Res. 1997;25:3389-402

28. Dohm JC, Minoche AE, Holtgräwe D, Capella-Gutiérrez S, Zakrzewski F, Tafer $\mathrm{H}$, et al. The genome of the recently domesticated crop plant sugar beet (Beta vulgaris). Nature. 2014;505:546-9

29. Goodstein DM, Shu S, Howson R, Neupane R, Hayes RD, Fazo J, et al. Phytozome: a comparative platform for green plant genomics. Nucleic Acids Res. 2012:40:1178-86.

30. Benson DA, Cavanaugh M, Clark K, Karsch-Mizrachi I, Lipman DJ, Ostell J, et al. GenBank. Nucleic Acids Res. 2013:41(Database issue):D36-42.

31. Huala E, Dickerman AW, Garcia-Hernandez M, Weems D, Reiser L, LaFond F, et al. The Arabidopsis information resource (TAIR): a comprehensive database and web-based information retrieval, analysis, and visualization system for a model plant. Nucleic Acids Res. 2001;29:102-5.

32. Apweiler R, Bairoch A, Wu CH, Barker WC, Boeckmann B, Ferro S, et al. UniProt: the universal protein knowledgebase. Nucleic Acids Res. 2004;32:D115-9.

33. Lightfoot DJ, Jarvis DE, Ramaraj T, Lee R, Jellen EN, Maughan PJ. Singlemolecule sequencing and hi-C-based proximity-guided assembly of amaranth (Amaranthus hypochondriacus) chromosomes provide insights into genome evolution. BMC Biol. 2017;15:1-15.

34. Berardini TZ, Mundodi S, Reiser L, Huala E, Garcia-Hernandez M, Zhang P, et al. Functional annotation of the Arabidopsis genome using controlled vocabularies. Plant Physiol. 2004:135:745-55.

35. Tuskan GA, DiFazio S, Jansson S, Bohlmann J, Grigoriev I, Hellsten U, et al. The genome of black cottonwood, Populus trichocarpa (Torr. \& Gray). 2003; 313:1596-605. 
36. Ming R, Hou S, Feng Y, Yu Q, Dionne-Laporte A, Saw JH, et al. The draft genome of the transgenic tropical fruit tree papaya (Carica papaya Linnaeus). Nature. 2008;452:991-6.

37. Harkess A, Zhou J, Xu C, Bowers JE, Van Der Hulst R, Ayyampalayam S, et al. The asparagus genome sheds light on the origin and evolution of a young Y chromosome. Nat Commun. 2017;8:1279.

38. Langmead B, Trapnell C, Pop M, Salzberg SL. Ultrafast and memory-efficient alignment of short DNA sequences to the human genome. Genome Biol. 2009;10:R25

39. Li B, Dewey CN. RSEM: accurate transcript quantification from RNA-Seq data with or without a reference genome. BMC Bioinformatics. 2011;12:323.

40. Robinson MD, Oshlack A. A scaling normalization method for differential expression analysis of RNA-seq data. Genome Biol. 2010;11:R25.

41. Robinson MD, McCarthy DJ, Smyth GK. edgeR: a Bioconductor package for differential expression analysis of digital gene expression data. Bioinformatics. 2009;26:139-40.

42. Wickham H. ggplot2: elegant graphics for data analysis. New York: SpringerVerlag; 2009.

43. Benjamini $Y$, Hochberg $Y$. Controlling the false discovery rate: a practical and powerful approach to multiple testing. J R Stat Soc Ser B. 1995;57:289300.

44. Fisher RA. The design of experiments. 8th edition. Edinburgh. London: Oliver and Boyd; 1966.

45. Bonferroni CE. II calcolo delle assicurazioni su gruppi di teste. Stud Onore del Profr Salvatore Ortu Carboni. 1935:13-60

46. Clarke K, Yang Y, Marsh R, Xie LL, Zhang KK. Comparative analysis of de novo transcriptome assembly. Sci China Life Sci. 2013;56:156-62.

47. Lander ES, Linton LM, Birren B, Nusbaum C, Zody MC, Baldwin J, et al. Initial sequencing and analysis of the human genome. Nature. 2001;409:860-921.

48. Meyerowitz EM, Somerville CR, editors. Arabidopsis. New York: Cold Spring Harbor Laboratory Press; 1994.

49. Harris M, Clark J, Ireland A, Lomax J, Ashburner M, Foulger R, et al. The gene ontology (GO) database and informatics resource. Nucleic Acids Res. 2004 32:D258-61.

50. Stewart CN, Tranel PJ, Horvath DP, Anderson JV, Rieseberg LH, Westwood $\mathrm{JH}$, et al. Evolution of weediness and invasiveness: charting the course for weed genomics. Weed Sci. 2009;57:451-62.

51. Basu C, Halfhill MD, Mueller TC, Stewart CN. Weed genomics: new tools to understand weed biology. Trends Plant Sci. 2004:9:391-8.

52. Owen MDK, Beckie HJ, Leeson JY, Norsworthy JK, Steckel LE. Integrated pest management and weed management in the United States and Canada. Pest Manag Sci. 2015;71:357-76.

53. Westwood JH, Charudattan R, Duke SO, Fennimore SA, Marrone P, Slaughter DC, et al. Weed management in 2050: perspectives on the future of weed science. Weed Sci. 2018:1-11.

54. Krochko JE, Abrams GD, Loewen MK, Abrams SR, Cutler AJ. (+)-abscisic acid 8'-hydroxylase is a cytochrome P450 monooxygenase. Plant Physiol. 1998; 118:849-60.

55. Okamoto M, Kuwahara A, Seo M, Kushiro T, Asami T, Hirai N, et al. CYP707A and CYP707A2, which encode abscisic acid 8'-hydroxylases, are indispensable for proper control of seed dormancy and germination in Arabidopsis. Plant Physiol. 2006;141:97-107.

56. Nambara E, Marion-Poll A. Abscisic acid biosynthesis and catabolism. Annu Rev Plant Biol. 2005;56:165-85.

57. Devine MD, Harren JC, Abrams SR, Gusta LV. Effect of drought stress, abscisic acid, and abscisic acid analogues on the efficacy of diclofop-methyl and tralkoxydim. J Plant Growth Regul. 1995;14:77-84.

58. Bigeard J, Colcombet J, Hirt H. Signaling mechanisms in pattern-triggered immunity (PTI). Mol Plant. 2015;8:521-39.

59. Kilian J, Whitehead D, Horak J, Wanke D, Weinl S, Batistic O, et al. The AtGenExpress global stress expression data set: protocols, evaluation and model data analysis of UV-B light, drought and cold stress responses. Plant J. 2007:50:347-63.

60. Ge Y, Li Y, Zhu YM, Bai X, Lv DK, Guo D, et al. Global transcriptome profiling of wild soybean (Glycine soja) roots under $\mathrm{NaHCO} 3$ treatment. BMC Plant Biol. 2010;10.

61. Johnson SM, Doherty SJ, Croy RRD. Biphasic superoxide generation in potato tubers. A self-amplifying response to stress. Plant Physiol. 2003;131 March:1440-1449.
62. Baxter A, Mittler R, Suzuki N. ROS as key players in plant stress signalling. J Exp Bot. 2014;65:1229-40.

63. Hamprecht G, Witschel M, Hawkes TR, Edmunds AJF, Morris JA, van Almsick A. Herbicides with bleaching properties. In: Krämer W, Schirmer U, Jeschke P. Witschel M, editors. Modern Crop Protection Compounds. Second. 2012. p. $197-276$.

64. Sandermann H. Plant metabolism of xenobiotics. Trends Biochem Sci. 1992; 17:82-4.

65. Bártíková H, Skálová L, Stuchíková L, Vokřál I, Vaněk T, Podlipná R. Xenobiotic-metabolizing enzymes in plants and their role in uptake and biotransformation of veterinary drugs in the environment. Drug Metab Rev. 2015;47:374-87.

66. Al-Whaibi MH. Plant heat-shock proteins: a mini review. J King Saud Univ Sci. 2011;23:139-50.

67. Babenko LM, Shcherbatiuk MM, Skaterna TD, Kosakivska IV. Lipoxygenases and their metabolites in formation of plant stress tolerance. Ukr Biochem J. 2017:89:5-21.

68. Bak S, Beisson F, Bishop G, Hamberger B, Höfer R, Paquette S, et al. Cytochromes P450. Arab B. 2011;9:e0144.

69. Chae EL, Ahn JH, Lim J. Molecular genetic analysis of tandemly located glycosyltransferase genes, UGT73B1, UGT73B2, and UGT73B3, in Arabidopsis thaliana. J Plant Biol. 2006:49:309-14.

70. Das M, Reichman JR, Haberer G, Welzl G, Aceituno FF, Mader MT, et al. A composite transcriptional signature differentiates responses towards closely related herbicides in Arabidopsis thaliana and Brassica napus. Plant Mol Biol. 2010:72:545-56

71. Szklarczyk D, Franceschini A, Wyder S, Forslund K, Heller D, Huerta-Cepas J, et al. STRING v10: protein-protein interaction networks, integrated over the tree of life. Nucleic Acids Res. 2015;43:D447-52.

72. Anthony RG, Reichelt S, Hussey PJ. Dinitroaniline herbicide-resistant transgenic tobacco plants generated by co-overexpression of a mutant alpha-tubulin and a beta-tubulin. Nat Biotechnol. 1999;17:712-6.

73. Pasquer F, Ochsner U, Zarn J, Keller B. Common and distinct gene expression patterns induced by the herbicides 2,4-dichlorophenoxyacetic acid, cinidon-ethyl and tribenuron-methyl in wheat. Pest Manag Sci. 2006; 62:1155-67

74. Jiang Z, Ma B, Erinle KO, Cao B, Liu X, Ye S, et al. Enzymatic antioxidant defense in resistant plant: Pennisetum americanum (L.) K. Schum during long-term atrazine exposure. Pestic Biochem Physiol. 2016;133:59-66.

75. Murgia I, Tarantino D, Vannini C, Bracale M, Carravieri S, Soave C. Arabidopsis thaliana plants overexpressing thylakoidal ascorbate peroxidase show increased resistance to paraquat-induced photooxidative stress and to nitric oxide-induced cell death. Plant J. 2004;38:940-53.

76. Matsunaka S, Ito K. Paraquat resistance in Japan. In: Caseley JC, Cussans GW, Atkin RK, editors. Herbicide Resistance in Weeds and Crops. ButterworthHeinemann; 1991. p. 77-86.

77. Krasensky J, Broyart C, Rabanal FA, Jonak C. The redox-sensitive chloroplast trehalose-6-phosphate phosphatase AtTPPD regulates salt stress tolerance. Antioxid Redox Signal. 2014;21:1289-304.

78. Kurepa J, Karangwa C, Duke LS, Smalle JA. Arabidopsis sensitivity to protein synthesis inhibitors depends on 265 proteasome activity. Plant Cell Rep. 2010;29:249-59.

Ready to submit your research? Choose BMC and benefit from

- fast, convenient online submission

- thorough peer review by experienced researchers in your field

- rapid publication on acceptance

- support for research data, including large and complex data types

- gold Open Access which fosters wider collaboration and increased citations

- maximum visibility for your research: over $100 \mathrm{M}$ website views per year

At $\mathrm{BMC}$, research is always in progress.

Learn more biomedcentral.com/submission 